\title{
Ising $n$-fold integrals as diagonals of rational functions and integrality of series expansions
}

March 17th, 2013

\author{
A. Bostan ${ }^{\llbracket}$, S. Boukraa||, G. Christol ${ }^{\ddagger}$, S. Hassani ${ }^{\S}$, J-M. \\ Maillard ${ }^{£}$ \\ ฯ INRIA, Bâtiment Alan Turing, 1 rue Honoré d'Estienne d'Orves, Campus de \\ l'École Polytechnique, 91120 Palaiseau, France \\ || LPTHIRM and Département d'Aéronautique, Université de Blida, Algeria \\ $¥$ Institut de Mathématiques de Jussieu, UPMC, Tour 25, 4ème étage, 4 Place \\ Jussieu, 75252 Paris Cedex 05, France \\ $\S$ Centre de Recherche Nucléaire d'Alger, 2 Bd. Frantz Fanon, B.P. 399, 16000 \\ Alger, Algeria \\ $£$ LPTMC, UMR 7600 CNRS, Université de Paris 6, Tour 23, 5ème étage, case \\ 121, 4 Place Jussieu, 75252 Paris Cedex 05, France
}

\begin{abstract}
We show that the $n$-fold integrals $\chi^{(n)}$ of the magnetic susceptibility of the Ising model, as well as various other $n$-fold integrals of the "Ising class", or $n$-fold integrals from enumerative combinatorics, like lattice Green functions, correspond to a distinguished class of functions generalising algebraic functions: they are actually diagonals of rational functions. As a consequence, the power series expansions of the, analytic at $x=0$, solutions of these linear differential equations "Derived From Geometry" are globally bounded, which means that, after just one rescaling of the expansion variable, they can be cast into series expansions with integer coefficients. We also give several results showing that the unique analytical solution of Calabi-Yau ODEs, and, more generally, Picard-Fuchs linear ODEs with solutions of maximal weights, are always diagonal of rational functions. Besides, in a more enumerative combinatorics context, generating functions whose coefficients are expressed in terms of nested sums of products of binomial terms can also be shown to be diagonals of rational functions. We finally address the question of the relations between the notion of integrality (series with integer coefficients, or, more generally, globally bounded series) and the modularity of ODEs.
\end{abstract}

This paper is the short version of the larger (100 pages) version [19], available on http://arxiv. org/abs/1211.6031, where all the detailed proofs are given and where a larger set of examples is displayed.

PACS: 05.50.+q, 05.10.-a, 02.30.Hq, 02.30.Gp, 02.40.Xx

AMS Classification scheme numbers: 34M55, 47E05, 81Qxx, 32G34, 34Lxx, $34 \mathrm{Mxx}, 14 \mathrm{Kxx}$

Key-words: Diagonals of rational functions, Hadamard products, series with integer coefficients, globally bounded series, differential equations Derived From Geometry, Hauptmoduls, modular forms, Calabi-Yau ODEs, modularity, modular 
polynomial, modular equation, nome, mirror maps, Yukawa coupling, Eisenstein constant, lattice Green functions. 


\section{Introduction}

The series expansions of many magnetic susceptibilities (or many other quantities, like the spontaneous magnetisation) of the Ising model on various lattices in arbitrary dimensions are actually series with integer coefficients $[1,2,3]$. This is a consequence of the fact that, in a van der Waerden type expansion of the susceptibility, all the contributing graphs are the ones with exactly two odd-degree vertices and the number of such graphs is an integer. When series expansions in theoretical physics, or mathematical physics, do not have such an obvious counting interpretation, the puzzling emergence of series with integer coefficients is a strong indication that some fundamental structure, symmetry, concept have been overlooked, and that a deeper understanding of the problem remains to be discoveredł. Algebraic functions are known to produce series with integer coefficients. Eisenstein's theorem [5] states that the Taylor series of a (branch of an) algebraic function can be recast into a series with integer coefficients, up to a rescaling by a constant (Eisenstein constant). An intriguing result due to Fatou [6] (see pp. 368-373) states that a power series with integer coefficients and radius of convergence (at least) one, is either rational, or transcendental. This result also appears in Pólya and Szegö's famous Aufgaben book [7] (see Problem VIII-167). Pólya [8] conjectured a stronger result, namely that a power series with integer coefficients which converges in the open unit disk is either rational, or admits the unit circle as a natural boundary (i.e. it has no analytic continuation beyond the unit disk). This was eventually proved 9 by Carlson [10]. Along this natural boundary line, it is worth recalling $[11,12,13,15,16]$ that the series expansions of the full magnetic susceptibility of the 2D Ising model [17] correspond to a power series with integer coefficients $\dagger$. For them, the unit circle certainly arises as a natural boundary [18] (with respect to the modulus variable $k$ ), but, unfortunately, this cannot be justified by Carlson's theorem $\dagger$.

A series with natural boundaries cannot be D-finite\#, i.e. solution of a linear differential equation with polynomial coefficients [22,23]. For simplicity, let us restrict to series with integer coefficients (or series that have integer coefficients up to a variable rescaling), that are series expansions of $\mathrm{D}$-finite functions. $\mathrm{Wu}, \mathrm{McCoy}$, Tracy and Barouch [24] have shown that the previous full magnetic susceptibility of the 2D Ising model can be expressed (up to a normalisation factor $(1-s)^{1 / 4} / s$, see $[13,25])$ as an infinite sum of $n$-fold integrals, denoted by $\tilde{\chi}^{(n)}$, which are actually $D$-finiteई. We found out that the corresponding (minimal order) differential operators are Fuchsian [11, 13], and, in fact, "special" Fuchsian operators: the critical exponents

$\ddagger$ The emergence of positive integer coefficients corresponds to the existence of some underlying measure [4].

ฯ The Pólya-Carlson result can be used to prove that some integer sequences, such as the sequence of prime numbers $\left(p_{n}\right)$ [9], do not satisfy any linear recurrence relation with polynomial coefficients. $\dagger$ In some variable $w[11,12,13,15]$. In the modulus variable $k$, one needs to perform a simple rescaling by a factor 2 or 4 according to the type of (high, or low temperature) expansions.

$\dagger+$ The radius of convergence is 1 with respect to the modulus variable $k$, in which the series does not have integer coefficients, being globally bounded only (this means that it can be recast into a series with integer coefficients by one rescaling of the variable $k$ ). If one considers the series expansion with respect to another variable (such as $w$ ) in which the series does have integer coefficients, then the radius of convergence is not 1 .

\# D-finite series are sometimes called holonomic. A priori, for multivariate functions, these notions differ [19]. The equivalence of these notions is proved by deep results of Bernšter̆n [20] and Kashiwara [21].

$\S$ For Ising models on higher dimensional lattices $[1,2,3]$ no such decomposition of susceptibilities, as an infinite sum of D-finite functions, should be expected at first sight. 
for all their singularities are rational numbers, and their Wronskians are $N$-th roots of rational functions [26]. Furthermore, it has been shown later that these $\tilde{\chi}^{(n)}$ 's are, in fact, solutions of globally nilpotent operators [27], or G-operators [28, 29]. It is worth noting that the series expansions, at the origin, of the $\tilde{\chi}^{(n)}$ 's, in a well-suited variable $[13,25] w$, actually have integer coefficients, even if this result does not have an immediate proof $\dagger$ for all integers $n$ (in contrast with the full susceptibility). From the first truncated series expansions of $\tilde{\chi}^{(n)}$, the coefficients for generic $n$ can be inferred [27]

$$
\begin{aligned}
& \tilde{\chi}^{(n)}(w)=2^{n} \cdot w^{n^{2}} \cdot\left(1+4 n^{2} \cdot w^{2}+2 \cdot\left(4 n^{4}+13 n^{2}+1\right) \cdot w^{4}\right. \\
& \quad+\frac{8}{3} \cdot\left(n^{2}+4\right)\left(4 n^{4}+23 n^{2}+3\right) \cdot w^{6} \\
& \quad+\frac{1}{3} \cdot\left(32 n^{8}+624 n^{6}+4006 n^{4}+8643 n^{2}+1404\right) \cdot w^{8} \\
& \left.\quad+\frac{4}{15} \cdot\left(n^{2}+8\right) \cdot\left(32 n^{8}+784 n^{6}+6238 n^{4}+16271 n^{2}+3180\right) \cdot w^{10}+\cdots\right) .
\end{aligned}
$$

Note that the coefficients of $\tilde{\chi}^{(n)}(w) / 2^{n}$, which depend on $n^{2}$, are integer coefficients when $n$ is any integer, this integrality property of the coefficients for any integer $n$ being not straightforward (see [19]). These coefficients are valid up to $w^{2}$ for $n \geq 3$, $w^{4}$ for $n \geq 5, w^{6}$ for $n \geq 7, w^{8}$ for $n \geq 9$, and $w^{10}$ for $n \geq 11$ (in particular it should be noted that $\tilde{\chi}^{(n)}$ is an even function of $w$ only for even $n$ ). Further studies on these $\tilde{\chi}^{(n)}$ 's showed the fundamental role played by the theory of elliptic functions (elliptic integrals, modular forms) and, much more unexpectedly, CalabiYau ODEs [30, 31]. These recent structure results thus suggest to see the occurrence of series with integer coefficients as a consequence of modularity [32] (modular forms, mirror maps [30, 31, 32, 33], etc) in the Ising model.

Along this line, many other examples of series with integer coefficients emerged in mathematical physics (differential geometry, lattice statistical physics, enumerative combinatorics, replicable functions $\sharp . .$. ). One must, of course, also recall Apéry's results [39]. Appendix A gives a list of modular forms, and their associated series with integer coefficients, corresponding to various lattice Green functions [40, 41, 42, 43], that are, often, expressed in terms of HeunG functions $\ddagger$ which can be written as hypergeometric functions with two alternative pullbacks (see also sections (6.1) and (6.2) below). Let us underline, in Appendix A, the Green function for the diamond lattice [43], the Green function for the face-centred cubic lattice (see equation (19) in [43]), and more examples corresponding to the spanning tree generating functions [44] (and Mahler measures). This integrality is also seen in the nome and in other quantities like the Yukawa coupling [30].

In this paper we restrict on series with integer coefficients, or, more generally, globally bounded [45] series of one complex variable, but it is clear that this integrality property does also occur in physics with several complex variables: they can, for

$\dagger$ We are interested in this paper in the emergence of integers as coefficients of D-finite series. In general, this emergence is not obvious: it cannot be simply explained at the level of the linear recurrence satisfied by the coefficients, as illustrated by the case of Apéry's calculations (see also section (6.2) and Appendix D).

I Which is not a surprise for Yang-Baxter integrability specialists.

$\sharp$ The concept of replicable functions is closely related to modular functions [34], (see the replicability of Hauptmoduls), Calabi-Yau threefolds, and more generally the concept of modularity [32, 35, 36, $37,38]$.

$\ddagger$ Generically HeunG functions are far from being modular forms. 
instance, be seen for the previous (D-finite $\S) n$-fold integrals $\tilde{\chi}^{(n)}$ for the anisotropic Ising model [46] (or for the Ising model on the checkerboard lattice), or on the example of the lattice Ising models with a magnetic field $\ddagger$ (see for instance, Bessis et al. [4]).

We take, here, a learn-by-example approach: on such quite technical questions one often gets a much deeper understanding from highly non-trivial examples than from sometimes too general, or slightly obfuscated, mathematical demonstrations.

The main result of the paper will be to show that the $\tilde{\chi}^{(n)}$ 's are globally bounded series, as a consequence of the fact that they are actually diagonals of rational functions for any value of the integer $n$. We will generalise these ideas, and show that an extremely large class of problems of mathematical physics can be interpreted in terms of diagonals of rational functions: $n$-fold integrals with algebraic integrand of a certain type that we will characterise, Calabi-Yau ODEs, MUM linear ODEs [48], series whose coefficients are nested sums of products of binomials, etc.

Another purpose of this paper is to "disentangle" the notion of series with integer coefficients (integrality) and the notion of modularity [32, 35, 36, 37, 38, 49, 50]. In this "down-to-earth" paper we essentially restrict to Picard-Fuchs ODEs and to a "CalabiYau" framework, therefore modularity $\sharp$ will just mean that the series solutions of Picard-Fuchs ODEs, as well as the corresponding nome series, and the Yukawa series, have integer coefficients.

The paper is organised as follows. Section (2) introduces the main concepts we need in this very paper, namely the concept of diagonals of rational or algebraic functions, and the concept of globally bounded series, recalling that diagonals of rational or algebraic functions are necessarily globally bounded series. Section (3) shows the main result of the paper, namely that the $n$-fold Ising integrals $\tilde{\chi}^{(n)}$ are diagonals of rational functions for any value of the integer $n$, the corresponding series being, thus, globally bounded. Section (4) shows that series with (nested sums of products of) binomials coefficients are diagonals of rational functions. Section (5) discusses, in the most general framework, the conjecture that D-finite globally bounded series could be necessarily diagonals of rational functions. Section (6) provides a set of modular forms examples (in particular lattice Green functions see Appendix A). Beyond modular forms, using new determinantal identities on the Yukawa couplings, and focusing on Hadamard products of modular forms, section (7) analyses the difference between integrality and modularity, showing that the two concepts are actually quite different. Section (8) addresses, more specifically, the Calabi-Yau modularity, and the difference between integrality and modularity, underlining that the integrality of the nome series is crucial for modularity, the integrality of the Yukawa series being not sufficient. The conclusion, section (9), emphasises the difference between the "special properties" of geometrical nature and the ones of arithmetic nature, emerging in theoretical physics. Several large appendices provide detailed examples illustrating pedagogically the previous sections. In particular Appendix A provides many modular forms examples associated with lattice Green functions, and Appendix E provides new representations of Yukawa couplings as ratios

$\S$ For several complex variables the ODEs of the paper are replaced by Picard-Fuchs systems.

$\ddagger$ Along this line, original alternative representations of the partition function of the Ising model in a magnetic field are also worth recalling [47].

\# Modularity is a wider concept than this "Calabi-Yau" modularity (see modular up to a Tate twist, modular Galois representations [51]). Modular forms provide the simplest examples (see Appendix A) of modularity (see also Serre's modularity conjecture, and the Taniyama-Shimura conjecture). For a first introduction to these ideas see [52]. 
of determinants.

\section{Series integrality, diagonal of rational functions}

Let us recall some concepts that will be fundamental in this paper, first the notion of globally bounded series, and, then, the concept of diagonal of a function 9 , and some of its most important properties. The main reason to introduce this concept of diagonal of function, not very familiar to physicists, is that it enables to consider diagonal of rational functions, this class of functions filling the gap between algebraic functions and $G$-series: they can be seen as generalisations of algebraic functions. Thus this class of functions can play a key role to decipher the complexity of functions occurring in theoretical physics.

\subsection{Globally bounded series}

Let us first recall the definition of being globally bounded [45] for a series. Consider a series expansion with rational coefficients, with non-zero radius of convergencet. The series is said to be globally bounded if there exists an integer $N$ such that the series can be recast into a series with integer coefficients with just one rescaling $x \rightarrow N x$.

A necessary condition for being globally bounded is that only a finite number of primes occur as factors of the denominators of the rational number series coefficients. There is also a condition on the growth of these denominators, that must be bounded exponentially [45], in such a way that the series has a non-zero $p$-adic radius of convergence for all primes $p$. When this is the case, it is easy to see that these series can be recast, with just one rescaling, into series with integer coefficients

\subsection{Definition of the diagonal of a rational function}

Assume that $\mathcal{F}\left(z_{1}, \ldots, z_{n}\right)=P\left(z_{1}, \ldots, z_{n}\right) / Q\left(z_{1}, \ldots, z_{n}\right)$ is a rational function, where $P$ and $Q$ are polynomials of $z_{1}, \cdots, z_{n}$ with rational coefficients such that $Q(0, \ldots, 0) \neq 0$. This assumption implies that $\mathcal{F}$ can be expanded at the origin as a Taylor series with rational number coefficients

$$
\mathcal{F}\left(z_{1}, z_{2}, \ldots, z_{n}\right)=\sum_{m_{1}=0}^{\infty} \cdots \sum_{m_{n}=0}^{\infty} F_{m_{1}, \ldots, m_{n}} \cdot z_{1}^{m_{1}} \cdots z_{n}^{m_{n}} .
$$

The diagonal of $\mathcal{F}$ is defined as the series of one variable

$$
\operatorname{Diag}\left(\mathcal{F}\left(z_{1}, z_{2}, \ldots, z_{n}\right)\right)=\sum_{m=0}^{\infty} F_{m, m, \ldots, m} \cdot z^{m} .
$$

More generally, one can define, in a similar way, the diagonal of any multivariate power series $\mathcal{F}$, with rational number coefficients, or with coefficients in a finite field $\ddagger$.

I The functions are in fact defined by series of several complex variables: they have to be Taylor, or Laurent, series (no Puiseux series).

$\dagger$ A series like the Euler-series $\sum_{n=0}^{\infty} n ! \cdot x^{n}$ which has integer coefficients is excluded.

$\sharp$ For a first set of series with integer coefficients, see Appendix A, where a set of such series with integer coefficients corresponding to modular forms is displayed. See also (6.1) and (6.2) below.

$\ddagger$ The definition even extends to multivariate Laurent power series, see e.g. [53]. 


\subsection{Main properties of diagonals}

The concept of diagonal of a function has a lot of interesting properties (see for instance [54]). Let us recall, through examples, some of the most important ones.

The study of diagonals goes back, at least, to Pólya [55], in a combinatorial context, and to Cameron and Martin [56] in an analytical context related to Hadamard products [57]. Pólya showed that the diagonal of a rational function in two variables is always an algebraic function. The most basic example is $\mathcal{F}=1 /\left(1-z_{1}-z_{2}\right)$, for which

$$
\begin{gathered}
\operatorname{Diag}(\mathcal{F})=\operatorname{Diag}\left(\sum_{m_{1}=0}^{\infty} \sum_{m_{2}=0}^{\infty}\left(\begin{array}{c}
m_{1}+m_{2} \\
m_{1}
\end{array}\right) \cdot z_{1}^{m_{1}} z_{2}^{m_{2}}\right) \\
=\sum_{m=0}^{\infty}\left(\begin{array}{c}
2 m \\
m
\end{array}\right) \cdot z^{m}=\frac{1}{\sqrt{1-4 z}} .
\end{gathered}
$$

The proof of Pólya's result is based on the simple observation that the diagonal $\operatorname{Diag}(\mathcal{F})$ is equal to the coefficient of $z_{1}^{0}$ in the expansion of $\mathcal{F}\left(z_{1}, z / z_{1}\right)$. Therefore, by Cauchy's integral theorem, $\operatorname{Diag}(\mathcal{F})$ is given by the contour integral

$$
\operatorname{Diag}(\mathcal{F})=\frac{1}{2 \pi i} \oint_{\gamma} \mathcal{F}\left(z_{1}, z / z_{1}\right) \frac{d z_{1}}{z_{1}},
$$

where the contour $\gamma$ is a small circle around the origin. Therefore, by Cauchy's residue theorem, $\operatorname{Diag}(\mathcal{F})$ is the sum of the residues of the rational function $\mathcal{G}=$ $\mathcal{F}\left(z_{1}, z / z_{1}\right) / z_{1}$ at all its singularities $s(z)$ with zero limit at $z=0$. Since the residues of a rational function of two variables are algebraic functions, $\operatorname{Diag}(\mathcal{F})$ is itself an algebraic function.

For instance, when $\mathcal{F}=1 /\left(1-z_{1}-z_{2}\right)$, then $\mathcal{G}=\mathcal{F}\left(z_{1}, z / z_{1}\right) / z_{1}$ has two poles at $s=\frac{1}{2}(1 \pm \sqrt{1-4 z})$. The only one approaching zero when $z \rightarrow 0$ is $s_{0}=\frac{1}{2}(1-\sqrt{1-4 z})$. If $p(s) / q(s)$ has a simple pole at $s_{0}$, then its residue at $s_{0}$ is $p\left(s_{0}\right) / q^{\prime}\left(s_{0}\right)$. Therefore

$$
\operatorname{Diag}(\mathcal{F})=\frac{1}{2 \pi i} \oint_{\gamma} \frac{d z_{1}}{z_{1}-z_{1}^{2}-z}=\frac{1}{1-2 s_{0}}=\frac{1}{\sqrt{1-4 z}} .
$$

\subsubsection{Diagonals of rational functions of more than two variables}

When passing from two to more variables, diagonalisation may still be interpreted using contour integration of a multiple complex integral over a so-called vanishing cycle [58]. However, the result is not an algebraic function anymore. A simple example is $\mathcal{F}=1 /\left(1-z_{2}-z_{3}-z_{1} z_{2}-z_{1} z_{3}\right)$, for which

$$
\operatorname{Diag}(\mathcal{F})=1+4 z+36 z^{2}+400 z^{3}+4900 z^{4}+63504 z^{5}+\cdots
$$

is equal to the complete elliptic integral of the first kind

$$
\operatorname{Diag}(\mathcal{F})=\sum_{m \geq 0}\left(\begin{array}{c}
2 m \\
m
\end{array}\right)^{2} \cdot z^{m}={ }_{2} F_{1}\left(\left[\frac{1}{2}, \frac{1}{2}\right],[1] ; 16 z\right),
$$

which is a transcendental function. A less obvious example (see [59] for a related example with a combinatorial flavor) is

$$
\begin{aligned}
\operatorname{Diag}\left(\frac{1}{1-z_{1}-z_{2}-z_{3}-z_{1} z_{2}-z_{2} z_{3}-z_{3} z_{1}-z_{1} z_{2} z_{3}}\right) \\
=\frac{1}{1-z} \cdot{ }_{2} F_{1}\left(\left[\frac{1}{3}, \frac{2}{3}\right],[1] ; \frac{54 z}{(1-z)^{3}}\right) .
\end{aligned}
$$


It was shown by Christol $[60,61,62]$ that the diagonal $\operatorname{Diag}(\mathcal{F})$ of any rational function $\mathcal{F}$ is $D$-finite, in the sense that it satisfies a linear differential equation with polynomial coefficients $\uparrow$. Moreover, the diagonal of any algebraic power series with rational coefficients is a $G$-function coming from geometry, i.e. it satisfies the Picard-Fuchs type differential equation associated with some one-parameter family of algebraic varieties. Diagonals of algebraic power series thus appear to be a distinguished class of $G$-functions\#. It will be seen below (see (2.5)) that algebraic functions with $n$ variables can be seen as diagonals of rational functions with $2 n$ variables. Thus diagonals of rational functions also appear to be a distinguished class of $G$-functions. It is worth noting that this distinguished class is stable by the Hadamard product: the Hadamard product of two diagonals of rational functions is the diagonal of rational function.

An immediate, but important property of diagonals of rational functions, with rational number coefficients, is that they are globally bounded, which means that they have integer coefficients up to a simple change of variable $z \rightarrow N z$, where $N \in \mathbb{Z}$.

\subsubsection{Diagonals of rational functions modulo primes}

Furstenberg [65] showed that the diagonal of any multivariate rational power series with coefficients in a field of positive characteristic is algebraic. Deligne $[58,53]$ extended this result to diagonals of algebraic functions. For instance, when $\mathcal{F}=$ $1 /\left(1-z_{2}-z_{3}-z_{1} z_{2}-z_{1} z_{3}\right)$, one gets modulo 7

$$
\begin{aligned}
\operatorname{Diag}(\mathcal{F}) \bmod 7 & =1+4 z+z^{2}+z^{3}+4 z^{7}+2 z^{8}+4 z^{9}+\cdots \\
& =\frac{1}{\sqrt[6]{1+4 z+z^{2}+z^{3}}} \bmod 7 .
\end{aligned}
$$

More generally, in this example, for any prime $p$, one has

$$
\operatorname{Diag}(\mathcal{F}) \quad=\quad P(z)^{1 /(1-p)} \quad \bmod p
$$

where the polynomial $P(z)$ is nothing, but $[66,67,68]$

$$
P(z)={ }_{2} F_{1}\left(\left[\frac{1}{2}, \frac{1}{2}\right],[1] ; 16 z\right)^{1-p} \bmod p=\sum_{n=0}^{(p-1) / 2}\left(\begin{array}{c}
p-1 / 2 \\
n
\end{array}\right)^{2} \cdot(16 z)^{n} .
$$

Note, however, that the Furstenberg-Deligne result [65, 58], that we illustrate, here, with $\mathcal{F}=1 /\left(1-z_{2}-z_{3}-z_{1} z_{2}-z_{1} z_{3}\right)$, goes far beyond the case of hypergeometric functions for which simple closed formulae can be displayed.

\subsection{Hadamard product and other products of series}

Let us also recall the notion of Hadamard product [57,69] of two series, that we will denote by a star.

$$
\begin{aligned}
& \text { If } \quad f(x)=\sum_{n=0}^{\infty} a_{n} \cdot x^{n}, \quad g(x)=\sum_{n=0}^{\infty} b_{n} \cdot x^{n}, \quad \text { then: } \\
& f(x) \star g(x)=\sum_{n=0}^{\infty} a_{n} \cdot b_{n} \cdot x^{n} .
\end{aligned}
$$

I A more general result was proved by Lipshitz [63]: the diagonal of any D-finite series is D-finite, see also [64].

\# Such diagonals are solutions of $G$-operators. They are functions that are always algebraic modulo any prime $p$. They fill the gap between algebraic functions and $G$-series: they can be seen as generalisations of algebraic functions. 
The notion of diagonal of a function and the notion of Hadamard product are obviously related:

$$
\operatorname{Diag}\left(f_{1}\left(x_{1}\right) \cdot f_{2}\left(x_{2}\right) \cdots f_{n}\left(x_{n}\right)\right)=f_{1}(x) \star f_{2}(x) \star \cdots \star f_{n}(x) .
$$

In other words, the diagonal of a product of functions with separate variables is equal to the Hadamard product of these functions in a common variable. In particular, the Hadamard product of $n$ rational (or algebraic, or even D-finite) power series is D-finite§.

The Hadamard product of two series with integer coefficients is straightforwardly a series with integer coefficients. Furthermore, the Hadamard product of two operators, annihilating two series, defined as the (minimal order, monic) linear differential operator annihilating the Hadamard product of these two series, is a product compatible with a large number of structures and concepts $\ddagger$ that naturally occur in lattice statistical mechanics. We have a similar compatibility property between the diagonal and the Hurwitz product [19, 70].

\subsection{Furstenberg's result on algebraic functions}

It was shown by Furstenberg [65] that any algebraic series in one variable can be written as the diagonal of a rational function of two variables. The basis of Furstenberg's result is the fact that if $f(x)$ is a power series without constant term, and is a root of a polynomial $P(x, y)$ such that $P_{y}(0,0) \neq 0$, then

$$
f(x)=\operatorname{Diag}\left(y^{2} \cdot \frac{P_{y}(x y, y)}{P(x y, y)}\right) \quad \text { where: } \quad P_{y}=\frac{\partial P}{\partial y} .
$$

When $P_{y}(0,0)=0$, formula (15) is not true anymore. However, Furstenberg's result still holds [19].

Note that this representation as diagonal of a rational function is, by no means unique, as can be seen on the algebraic function $\dagger$

$$
f=\frac{x}{\sqrt{1-x}}=x+\frac{1}{2} x^{2}+\frac{3}{8} x^{3}+\frac{5}{16} x^{4}+\frac{35}{128} x^{5}+\frac{63}{256} x^{6}+\cdots
$$

which is the diagonal of $(2 x y-c x+c y) /(x+y+2)$ for any rational number $c$.

Furstenberg's proof does not necessarily produce the simplest rational function (see [19]).

Furstenberg's result has been generalised to power series expansions of algebraic functions in an arbitrary number of variables n: any algebraic power series with rational coefficients is the diagonal of a rational function with $2 n$ variables (see Denef and Lipshitz [71]).

\section{Selected $n$-fold integrals are diagonals of rational functions}

Among many multiple integrals that are important in various domains of mathematical physics, and before considering other $n$-fold integrals of the "Ising class $\dagger \dagger$ ", let us first

$\S$ The Hadamard product of rational power series is still rational, but the Hadamard product of algebraic series is in general transcendental.

$\ddagger$ For instance, the Hadamard product of two globally nilpotent [27] operators is also globally nilpotent.

$\dagger$ Here, $f$ is annihilated by $P(x, y)=(1-x) y^{2}-x^{2}$, which is precisely such that $P_{y}(0,0)=0$.

I In the one-variable case, Puiseux series could be considered but only after ramifying the variable. †† Using the terminology introduced by Bailey et al. [14], see also [15]. 
consider the $n$-particle contribution to the magnetic susceptibility of the Ising model which we denote $\tilde{\chi}^{(n)}(w)$. They are given by $(n-1)$-dimensional integrals [11, 72]:

$$
\tilde{\chi}^{(n)}(w)=\frac{(2 w)^{n}}{n !}\left(\prod_{j=1}^{n-1} \int_{0}^{2 \pi} \frac{d \Phi_{j}}{2 \pi}\right) \cdot Y \cdot \frac{1+X}{1-X} \cdot X^{n-1} \cdot G,
$$

where, defining $\Phi_{0}$ by $\sum_{i=0}^{n-1} \Phi_{i}=0$, we set

$$
\begin{aligned}
& X=\prod_{i=0}^{n-1} x_{i}, \quad x_{i}=\frac{2 w}{A_{i}+\sqrt{A_{i}^{2}-4 w^{2}}}, \quad Y=\prod_{i=0}^{n-1} y_{i}, \quad y_{i}=\frac{1}{\sqrt{A_{i}^{2}-4 w^{2}}}, \\
& G=\prod_{0 \leq i<j \leq n-1} \frac{2-2 \cos \left(\Phi_{i}-\Phi_{j}\right)}{\left(1-x_{i} x_{j}\right)^{2}}, \quad \text { where: } \quad A_{i}=1-2 w \cos \left(\Phi_{i}\right) .
\end{aligned}
$$

The integrality property (1) had been checked [12] for the first $\tilde{\chi}^{(n)}$ 's and inferred [27] for generic $n$. We are going to prove it for any integer $n$, showing a much fundamental result, namely that all the $(n-1)$-fold integrals $\tilde{\chi}^{(n)}$ 's are very special: they are actually diagonals of rational functions.

\section{1. $\tilde{\chi}^{(3)}$ as a toy example}

At first sight the $\tilde{\chi}^{(n)}$ 's are involved transcendental holonomic functions. Could it be possible that they correspond to the distinguished class [53] of $G$-functions, generalising algebraic functions, which have an interpretation as diagonals of multivariate algebraic functions (and consequently diagonals of rational functions with twice more variables)? If this is the case, then the series of the $\tilde{\chi}^{(n)}$ 's must necessarily reduce modulo any prime to an algebraic function (see (2.3.2)). The $\tilde{\chi}^{(1)}$ and $\tilde{\chi}^{(2)}$ contributions being too degenerate (a rational function and a too simple elliptic function), let us consider the first non-trivial case, namely $\tilde{\chi}^{(3)}$. Its series expansion has already been displayed in [11]. It reads $\tilde{\chi}^{(3)} / 8=w^{9} \cdot F(w)$ with:

$$
F(w)=1+36 w^{2}+4 w^{3}+884 w^{13}+196 w^{5}+18532 w^{6}+6084 w^{7}+\cdots
$$

Since we have obtained the exact ODE satisfied by $\tilde{\chi}^{(3)}$ we can produce as many coefficients as we want in its series expansion. Let us consider this series modulo the prime $p=2$. It now reads the lacunary series

$$
F(w) \bmod 2=1+w^{8}+w^{24}+w^{56}+w^{120}+w^{248}+w^{504}+w^{1016}+\cdots,
$$

solution of the functional equations on $F(w)$ or, with $z=w^{8}$, on $G(z)=$ $1+w^{8} \cdot F(w)$

$$
F(w)=1+w^{8} \cdot F\left(w^{2}\right), \quad G(z)=z+G\left(z^{2}\right)
$$

where one recognises, with equation $G(z)=z+G\left(z^{2}\right)$, Furstenberg's example [65] of the simplest algebraic function in characteristic $2 \S$. In fact $H(w)=w^{9} F(w)$ is solution of the quadratic equation:

$$
H(w)^{2}+w \cdot H(w)+w^{10}=0 \quad \bmod 2 .
$$

$\ddagger$ For the $\tilde{\chi}^{(n)}$, the rescaling factor ("Eisenstein constant") is 2 or 4 according to the fact that one considers high or low temperature series $[12,26]$.

$\S$ Modulo the prime $p=2$, the previous functional equation becomes $G(z)=z+G(z)^{2}$. 
The calculations are more involved modulo $p=3$. Indeed, $H(w)=\tilde{\chi}^{(3)}(w) / 8$ satisfies, modulo 3 , the polynomial equation of degree nine

$$
\begin{aligned}
p_{9} \cdot H(w)^{9}+w^{6} \cdot p_{3} \cdot H(w)^{3} & +w^{10} \cdot p_{1} \cdot H(w) \\
& +w^{19} \cdot p_{0}^{(1)} \cdot p_{0}^{(2)}=0
\end{aligned}
$$

where:

$$
\begin{aligned}
p_{9}= & (w+1)^{3}\left(w^{2}+1\right)^{18}(w-1)^{24} \\
p_{3}= & \left(w^{2}+1\right)^{18}(1-w)^{15}\left(w^{4}-w^{2}-1\right), \quad p_{1}=\left(w^{2}+1\right)^{20}(1-w)^{13} \\
p_{0}^{(1)}= & w^{6}+w^{5}+w^{4}-w^{2}-w+1 \\
p_{0}^{(2)}= & w^{37}-w^{36}+w^{35}-w^{33}+w^{31}-w^{30}+w^{28}+w^{27}+w^{24}-w^{23}+w^{22} \\
& \quad-w^{21}-w^{18}-w^{16}+w^{14}-w^{12}-w^{11}-w^{10}+w^{7}-w^{5}-w^{3}-1
\end{aligned}
$$

The calculations are even more involved modulo larger primes. The (minimal order) linear differential operator annihilating the $\tilde{\chi}^{(3)}$ series mod. 5, reads $\dagger$ :

$$
\begin{gathered}
(x+1)\left(x^{2}+x+1\right)(x+2) \cdot x^{4} \cdot D_{x}^{4}+2 x^{3} \cdot\left(x^{3}+2 x^{2}+4 x+4\right)(x+4) \cdot D_{x}^{3} \\
+x^{2} \cdot\left(x^{4}+3 x^{3}+4\right) \cdot D_{x}^{2}+4 \cdot\left(x^{4}+3\right) \cdot x \cdot D_{x}+3
\end{gathered}
$$

If one can easily get this linear differential operator, finding the minimal polynomial of $\tilde{\chi}^{(3)}$ modulo 5 , generalising (20) or (21), or rather, the polynomial $\tilde{P}(\kappa, w)$, where $\kappa=\tilde{\chi}^{(3)}(w) / w^{9}$, such that $\tilde{P}(\kappa, w)=0$ mod. 5 , requires a very large number of coefficients. The polynomial [19] $\tilde{P}(\kappa, w)$ is of degree 50 in $\kappa$, of degree 832 in $x$ and is the sum of 4058 monomials.

One can imagine, in a first step, that the $\tilde{\chi}^{(3)}$ series mod. any prime $p$ are also algebraic functions, and, in a second step, that $\tilde{\chi}^{(3)}$ may be the diagonal of a rational function. In fact we are going to show, in the next section, a stronger result: the $\tilde{\chi}^{(n)}$ 's are actually diagonals of rational functions, for any integer $n$.

\subsection{The $\tilde{\chi}^{(n)}$ 's are diagonals of rational functions}

Let us, now, consider the general case where $n$ is an arbitrary integer. With the change of variable $z_{k}=\exp \left(i \Phi_{k}\right)$ (where $i^{2}=-1$ ), one clearly gets

$$
\begin{gathered}
\prod_{k=0}^{n-1} z_{k}=1, \quad \frac{d z_{j}}{z_{j}}=i d \Phi_{j}, \\
2 \cos \left(\Phi_{k}\right)=z_{k}+\frac{1}{z_{k}},
\end{gathered}
$$

and (17) becomes

$$
\tilde{\chi}^{(n)}(w)=\frac{(2 w)^{n}}{n !}\left(\prod_{j=1}^{n-1} \frac{1}{2 i \pi} \oint_{\mathcal{C}} \frac{d z_{j}}{z_{j}}\right) \cdot F\left(w, z_{1}, \ldots, z_{n-1}\right),
$$

where $\mathcal{C}$ is the path "turning once counterclockwise around the unit circle" and where $F$ is algebraic over $\mathbb{Q}\left(w, z_{1}, \ldots, z_{n-1}\right)$ and reads:

$$
F\left(w, z_{1}, \ldots, z_{n-1}\right)=Y \cdot X^{n-1} \cdot \frac{1+X}{1-X} \cdot G .
$$

$\dagger$ This operator is of zero 5-curvature [27]. 
Now, let us suppose that $F$ is analytic $\dagger$ at the origin, namely that it has a Taylor expansion (2). Then applying $(n-1)$ times the residue formula, one finds

$$
\tilde{\chi}^{(n)}(w)=\operatorname{Diag}\left(\frac{\left(2 z_{0} \cdots z_{n-1}\right)^{n}}{n !} \cdot F\left(z_{0} \cdots z_{n-1}, z_{1}, \ldots, z_{n-1}\right)\right) .
$$

To check that this is actually true, we introduce an auxiliary set, namely $\mathcal{T}_{n}$ the subset of Laurent series $\mathbb{Q}\left[z_{1}, \ldots, z_{n-1}, z_{1}^{-1}, \ldots, z_{n-1}^{-1}\right][[w]]$, consisting of series

$$
f\left(w, z_{1}, \ldots, z_{n-1}\right)=\sum_{m=0}^{\infty} P_{m} \cdot w^{m},
$$

where $P_{m}$ belongs to $\mathbb{Q}\left[z_{1}, \ldots, z_{n-1}, z_{1}^{-1}, \ldots, z_{n-1}^{-1}\right]$ and is such that the degree of $P_{m}$, in each of the $z_{k}^{-1}$, is at most $m$.

Then to prove that $F\left(z_{0} z_{1} \ldots z_{n-1}, z_{1}, \ldots, z_{n-1}\right)$ has a Taylor expansion, we only have to verify that $F\left(w, z_{1}, \ldots, z_{n-1}\right)$ belongs to this auxiliary set $\mathcal{T}_{n}$. Checking this is a straightforward step-by-step computation on auxiliary functions:

$$
\begin{aligned}
& A_{k}=1-w \cdot\left(z_{k}+\frac{1}{z_{k}}\right), \quad \quad \text { for: } \quad 1 \leq i \leq n-1, \\
& A_{k}=1-w \cdot\left(\frac{1}{z_{1} \cdots z_{n-1}}+z_{1} \cdots z_{n-1}\right), \quad \text { for: } \quad i=0 .
\end{aligned}
$$

Hence $A_{k}$ belongs to this auxiliary set $\mathcal{T}_{n}$. So to be sure that the inverse or the square root of some function in this auxiliary set $\mathcal{T}_{n}$ is also in this auxiliary set $\mathcal{T}_{n}$ we only have to check that its first Taylor coefficient is actually 1 , or $w, w^{2}$, or $w^{n}$, $n$ integer. It is straightforward to see that:

$$
\begin{aligned}
& A_{k}^{2}-4 w^{2}=1-2 w \cdot\left(z_{k}+\frac{1}{z_{k}}\right)+w^{2} \cdot\left(z_{k}-\frac{1}{z_{k}}\right)^{2}, \\
& \text { hence: } \quad \sqrt{A_{k}^{2}-4 w^{2}}=1+\cdots \text {, } \\
& y_{k}=\frac{1}{\sqrt{A_{k}^{2}-4 w^{2}}}=1+\cdots, \quad Y=1+\cdots, \\
& x_{k}=\frac{2 w}{A_{k}+\sqrt{A_{k}^{2}-4 w^{2}}}=w+\cdots, \quad x_{k} x_{j}=w^{2}+\cdots, \\
& X=w^{n}+\cdots, \quad \frac{1+X}{1-X}=1+\cdots, \\
& G=\prod_{0 \leq k<j \leq n-1}\left(\frac{-\left(z_{k}-z_{j}\right)^{2}}{\left(1-x_{k} x_{j}\right)^{2} \cdot z_{k} z_{j}}\right)=\prod_{0 \leq k<j \leq n-1}\left(\frac{-\left(z_{k}-z_{j}\right)^{2}}{z_{k} z_{j}}\right)+\cdots
\end{aligned}
$$

Thus, this shows that $F$ belongs to the auxiliary set $\mathcal{T}_{n}$ :

$$
\begin{aligned}
& F\left(w, z_{1}, \ldots, z_{n-1}\right)= \\
& \sum_{m=0}^{\infty} \sum_{m_{1}=-m}^{M_{1}(m)} \ldots \sum_{m_{n-1}=-m}^{M_{n-1}(m)} a_{m, m_{1}, \ldots, m_{n-1}} \cdot w^{m} \cdot z_{1}^{m_{1}+m} \ldots z_{n-1}^{m_{n-1}+m} .
\end{aligned}
$$

$\dagger$ One could consider Laurent, instead of Taylor, expansions, but this is a slight generalisation [53, $73,74]$. 
Consequently, it makes sense to take its diagonal. The residue theorem requires searching the terms not containing $z_{j}$ i.e. such as $m_{1}=\ldots=m_{n-1}=0$. One therefore gets:

$$
\tilde{\chi}^{(n)}=\sum_{m=0}^{\infty} a_{m, 0, \ldots, 0} \cdot w^{m} .
$$

In particular, we find

$$
\begin{aligned}
\tilde{\chi}^{(n)} & =\operatorname{Diag}\left(\sum_{m=0}^{\infty} \sum_{m_{1}=-m}^{M_{1}(m)} \ldots \sum_{m_{n-1}=-m}^{M_{n-1}(m)} a_{m, m_{1}, \ldots, m_{n-1}} \cdot z_{0}^{m} z_{1}^{m_{1}+m} \ldots z_{n-1}^{m_{n}-1+m}\right) \\
& =\operatorname{Diag}\left(F\left(z_{0} z_{1} \ldots z_{n-1}, z_{1}, \ldots, z_{n-1}\right)\right) .
\end{aligned}
$$

As $F$ is algebraic, $\tilde{\chi}^{(n)}$ is the diagonal of an algebraic function of $n$ variables and, consequently, the diagonal of a rational function of $2 n$ variables.

We thus see that we can actually find explicitly the algebraic function such that its diagonal is the $n$-fold integrals $\widetilde{\chi}^{(n)}$ : it is nothing but the integrand of the $n$-fold integral, up to simple transformations (namely $F\left(w, z_{1}, \ldots, z_{n-1}\right) \rightarrow$ $\left.F\left(z_{0} z_{1} \ldots z_{n-1}, z_{1}, \ldots, z_{n-1}\right)\right)$.

Remark: $\tilde{\chi}^{(n)}$ is a solution of a linear differential equation, and has a radius of convergence equal to $1 / 4$ in $w$. Among the other solutions of this equation, there is the function obtained by changing the square root appearing in $x_{k}$ into its opposite. A priori there are $2^{n}$ ways to do this, hence $2^{n}$ new solutions but, not all distinct. At first sight, for these new solutions, the $x_{k}$ 's are no longer in $\mathcal{T}_{n}$.

In fact, we find some quite interesting structure. Let us consider, for instance, the case of $\tilde{\chi}^{(3)}$. If one considers other choices of sign in front of the nested square roots in the integrand, the series expansions of the corresponding $n$-fold integrals read:

$$
\begin{gathered}
w+6 w^{2}+28 w^{3}+124 w^{4}+536 w^{5}+2280 w^{6}+9604 w^{7}+40164 w^{8} \\
+167066 w^{9}+692060 w^{10}+2857148 w^{11}+\cdots \\
w^{2}+6 w^{3}+30 w^{4}+140 w^{5}+628 w^{6}+2754 w^{7}+11890 w^{8}+50765 w^{9} \\
+214958 w^{10}+904286 w^{11}+\cdots
\end{gathered}
$$

One does remark that all these alternative series are, as $\tilde{\chi}^{(3)}$, series with integer coefficients [19].

\subsection{More $n$-fold integrals of the Ising class and a simple integral of the Ising class}

It is clear that the demonstration we have performed on the $\chi^{(n)}$ 's can also be performed straightforwardly, mutatis mutandis, with other $n$-fold integrals of the "Ising class $\ddagger[14,15]$ " like the $n$-fold integrals $\Phi_{H}$ in [72], which amounts to getting rid of the fermionic term $G$ (see (26)), the $\chi_{d}^{(n)}$ 's corresponding to $n$-fold integrals associated with the diagonal $\nmid$ susceptibility $[16,31]$ (the magnetic field is located on a diagonal of the square lattice), the $\Phi_{D}^{(n)}$ 's in [15] which are simple integrals, and also for all the lattice Green functions displayed in $[43,48]$, and the list is far from being

$\ddagger$ For the purpose of this section $n$-fold integrals of the "Ising class" will mean $n$-fold integrals that are known to arise in the study of the two-dimensional Ising model susceptibility.

$\dagger$ Of course this "diagonal $[16,31]$ wording" should not be confused with the notion of diagonal of a function. 
exhaustive. For instance, the simple integral $\Phi_{D}^{(n)}$ is the diagonal of the algebraic function:

$$
\begin{aligned}
& \frac{2}{n !} \cdot\left(1-t^{2}\right)^{-1 / 2} \cdot \frac{G_{n} F_{n}^{n-1}}{G_{n} F_{n}^{n-1}-(2 w t)^{n}}-\frac{1}{n !}, \quad \text { where: } \\
& F_{n}=\begin{array}{lll}
1 & -2 w+\left(1-4 w+4 w^{2}-4 w^{2} t^{2}\right)^{1 / 2} \\
G_{n}= & 1-2 w t \cdot T_{n-1}\left(\frac{1}{t}\right)+\left(\left(1-2 w t \cdot T_{n-1}\left(\frac{1}{t}\right)\right)^{2}-4 w^{2} \cdot t^{2}\right)^{1 / 2},
\end{array}
\end{aligned}
$$

and where $T_{n-1}(t)$ is the $(n-1)$-th Chebyshev polynomial of the first kind. The way we have obtained these Chebyshev results (32) is displayed in [19].

As opposed to the $\chi^{(n)}$ 's, the integral $\Phi_{D}^{(n)}(w)$ is the diagonal of an algebraic function of two variables (and, thus, the diagonal of a rational function of four variables) independently of the actual value of $n$.

If the $\chi^{(n)}$ 's are fundamental to understand the Ising model [17, 24], or the $\chi_{d}^{(n)}$ 's have a physical meaning associated with the diagonal susceptibility [16, 31] for the Ising model, most of the $n$-fold integrals of the "Ising class [14]" do not have that importance, or even that physical meaning (even if they have played a crucial role to understand the singularities of the Ising model [15]). What we see here with, for instance, the $\Phi_{H}$ 's $[15,72]$, is that the demonstration they are diagonal of rational functions is exactly the same as for the $\chi^{(n)}$ 's (see section 3.2), because of a key analyticity assumption of the integrand is also fulfilled.

\subsection{More general $n$-fold integrals as diagonals}

More generally the demonstration we have performed on the $\tilde{\chi}^{(n)}$ 's can be performed for any $n$-fold integral that can be recast in the following form:

$$
\int_{C} \int_{C} \cdots \int_{C} \frac{d z_{1}}{z_{1}} \frac{d z_{2}}{z_{2}} \cdots \frac{d z_{n}}{z_{n}} \cdot \mathcal{A}\left(x, z_{1}, z_{2}, \cdots, z_{n}\right),
$$

where the subscript $C$ denotes the unit circle, and where $\mathcal{A}$ denotes an algebraic function of the $n$ variables, which (this is the crucial ingredient), as a function of several variables $x$ and the $z_{k}$ 's, has an analytical expansion at $\left(x, z_{1}, z_{2}, \cdots, z_{n}\right)=$ $(0,0,0, \cdots, 0)$ :

$$
\begin{aligned}
& \mathcal{A}\left(x, z_{1}, z_{2}, \cdots, z_{n}\right)= \\
& \quad \sum_{m=0}^{\infty} \sum_{m_{1}=0}^{\infty} \sum_{m_{2}=0}^{\infty} \cdots \sum_{m_{n}=0}^{\infty} A_{m, m_{1}, m_{2}, \cdots, m_{n}} \cdot z_{1}^{m_{1}} z_{2}^{m_{2}} \cdots z_{n}^{m_{n}} \cdot x^{m} .
\end{aligned}
$$

Consequently, an extremely large set of $n$-fold integrals occurring in theoretical physics (lattice statistical mechanics, enumerative combinatorics, number theory, differential geometry, ...) can actually be seen to be diagonals of rational functions. These $n$-fold integrals correspond to series expansions (in the variation parameter $x$ ) that are globally bounded (can be written after one rescaling into series with integer coefficients), and are solutions of globally nilpotent [27] linear differential operators.

Such a general $n$-fold integral is, thus, the diagonal of an algebraic function (or of a rational function with twice more variables [71]) which is essentially the integrand of such $n$-fold integral. Furthermore, such a general $n$-fold integral is solution of a 
(globally nilpotent [27]) linear differential operator, that can be obtained exactly from the integrand, using the creative telescoping method [19].

Finally, in the case of Calabi-Yau ODEs (see below), these functions can be interpreted as periods of Calabi-Yau varieties, these algebraic varieties being essentially the integrand of such $n$-fold integrals. The integrand is thus the key ingredient to wrap, in the same bag, the algebraic geometry viewpoint, the differential geometry viewpoint, and the analytic and arithmetic approaches (series with integer coefficients).

\section{Calabi-Yau ODEs solutions and series with binomials seen as diagonals}

\subsection{Recalling Calabi-Yau ODEs}

Calabi-Yau ODEs have been defined in [75] as order-four linear differential ODEs that satisfy the following conditions: they are maximal unipotent monodromy $[76,77]$ (MUM), they satisfy a "Calabi-Yau condition" which amounts to imposing that the exterior squares of these order-four operators are of order five (instead of the order six one expects in the generic case), the series solution, analytic at $x=0$, is globally bounded (can be reduced to integer coefficients), the series of their nome and Yukawa coupling are globally bounded. In the literature, one finds also a cyclotomic condition on the monodromy at the point at $\infty, x=\infty$, and/or the conifold $\dagger$ character of one of the singularities [79].

Let us recall that a linear ODE has MUM (maximal unipotent monodromy [30, 78]) if all the exponents at (for instance) $x=0$ are zero. In a hypergeometric framework the MUM condition amounts to restricting to hypergeometric functions of the type ${ }_{n+1} F_{n}\left(\left[a_{1}, a_{2}, \cdots a_{n}\right],[1,1, \cdots 1], x\right)$, since the indicial exponents at $x=0$ are the solutions of $\rho\left(\rho+b_{1}-1\right) \cdots\left(\rho+b_{n}-1\right)=\rho^{n+1}=0$, where the $b_{j}$ are the lower parameters which are here all equal to 1 .

Let us consider a MUM order-four linear differential operator. The four solutions $y_{0}, y_{1}, y_{2}, y_{3}$ of this order-four linear differential operator read:

$$
\begin{aligned}
& y_{0}, \quad y_{1}=y_{0} \cdot \ln (x)+\tilde{y}_{1}, \quad y_{2}=y_{0} \cdot \frac{\ln (x)^{2}}{2}+\tilde{y}_{1} \cdot \ln (x)+\tilde{y}_{2}, \\
& y_{3}=y_{0} \cdot \frac{\ln (x)^{3}}{6}+\tilde{y}_{1} \cdot \frac{\ln (x)^{2}}{2}+\tilde{y}_{2} \cdot \ln (x)+\tilde{y}_{3},
\end{aligned}
$$

where $y_{0}, \tilde{y}_{1}, \tilde{y}_{2}, \tilde{y}_{3}$ are analytical at $x=0$ (with also $\left.\tilde{y}_{1}(0)=\tilde{y}_{2}(0)=\tilde{y}_{3}(0)=0\right)$.

The nome of this linear differential operator reads:

$$
q(x)=\exp \left(\frac{y_{1}}{y_{0}}\right)=x \cdot \exp \left(\frac{\tilde{y}_{1}}{y_{0}}\right) .
$$

Calabi-Yau ODEs have been defined as being MUM, thus having one solution analytical at $x=0$. As far as Calabi-Yau ODEs are concerned, the fact that this solution analytical at $x=0$ has an integral representation, and, furthermore, an integral representation of the form (34) together with (35), is far from clear, even if one may have a "Geometry-prejudice" that this solution, analytical at $x=0$, can be interpreted as a "Period" and "Derived From Geometry" [28, 29, 80].

Large tables of Calabi-Yau ODEs have been obtained by Almkvist et al. [78, $81,82]$. It is worth noting that the coefficients $A_{n}$ of the series corresponding to

$\dagger$ The local exponents are $0,1,1,2$. For the cyclotomic condition on the monodromy at $\infty$, see Proposition 3 in [78]. 
the solution analytical at $x=0$, are, most of the time, nested sums of product of binomials, less frequently nested sums of product of binomials and of harmonic numbers $\uparrow H_{n}$, and, in rare cases, no "closed formula" is known for these coefficients.

Let us show, in the case of $A_{n}$ coefficients being nested sums of product of binomials, that the solution of the Calabi-Yau ODE, analytical at $x=0$, which is by construction a series with integer coefficients, is actually a diagonal of rational function, and furthermore, that this rational function can actually be easily built.

\subsection{Calculating the rational function for nested product of binomials}

For pedagogical reasons we will just consider, here, a very simple example§ of a series $\mathcal{S}(x)$, with integer coefficients, given by a sum of product of binomials

$$
\begin{aligned}
\mathcal{S}(x) & =\sum_{n=0}^{\infty} \sum_{k=0}^{n}\left(\begin{array}{l}
n \\
k
\end{array}\right)^{3} \cdot x^{n}=\operatorname{HeunG}(-1 / 8,1 / 4,1,1,1,1 ;-x) \\
& =1+2 x+10 x^{2}+56 x^{3}+346 x^{4}+2252 x^{5}+15184 x^{6}+\cdots
\end{aligned}
$$

This is the generating function of sequence $\mathbf{A}$ in Zagier's tables of binomial coefficients sums (see p. 354 in [83]).

The calculations of this section can straightforwardly (sometimes tediously) be generalised to more complicated [84] nested sums of product of binomials $\dagger$.

Finding that a series is a diagonal of a rational function amounts to framing it

into a residue form like (34). In order to achieve this, we write the binomial $\left(\begin{array}{l}n \\ k\end{array}\right)$ as the residue

$$
\left(\begin{array}{l}
n \\
k
\end{array}\right)=\frac{1}{2 i \pi} \cdot \int_{C} \frac{(1+z)^{n}}{z^{k}} \cdot \frac{d z}{z},
$$

and, thus, we can rewrite $\mathcal{S}(x)$ as

$$
\begin{aligned}
& (2 i \pi)^{3} \cdot \mathcal{S}(x)= \\
& =\sum_{n=0}^{\infty} \iiint \sum_{k=0}^{n} \frac{1}{\left(z_{1} z_{2} z_{3}\right)^{k}} \cdot\left(\left(1+z_{1}\right)\left(1+z_{2}\right)\left(1+z_{3}\right) \cdot x\right)^{n} \cdot \frac{d z_{1} d z_{2} d z_{3}}{z_{1} z_{2} z_{3}} \\
& =\iiint \sum_{n=0}^{\infty} \frac{1-\left(1 /\left(z_{1} z_{2} z_{3}\right)\right)^{(n+1)}}{1-\left(1 /\left(z_{1} z_{2} z_{3}\right)\right)} \cdot\left(\left(1+z_{1}\right)\left(1+z_{2}\right)\left(1+z_{3}\right) \cdot x\right)^{n} \cdot \frac{d z_{1} d z_{2} d z_{3}}{z_{1} z_{2} z_{3}} \\
& =\quad-\iiint \sum_{n=0}^{\infty} \frac{z_{1} z_{2} z_{3}}{1-z_{1} z_{2} z_{3}} \cdot\left(\left(1+z_{1}\right)\left(1+z_{2}\right)\left(1+z_{3}\right) \cdot x\right)^{n} \cdot \frac{d z_{1} d z_{2} d z_{3}}{z_{1} z_{2} z_{3}} \\
& \quad+\iiint \sum_{n=0}^{\infty} \frac{1}{1-z_{1} z_{2} z_{3}} \cdot\left(\frac{\left(1+z_{1}\right)\left(1+z_{2}\right)\left(1+z_{3}\right) \cdot x}{z_{1} z_{2} z_{3}} \cdot \frac{d z_{1} d z_{2} d z_{3}}{z_{1} z_{2} z_{3}}\right. \\
& =\quad \iiint R\left(x ; z_{1}, z_{2}, z_{3}\right) \cdot \frac{d z_{1} d z_{2} d z_{3}}{z_{1} z_{2} z_{3}},
\end{aligned}
$$

T The generating function of Harmonic numbers is $H(x)=\sum H_{n} \cdot x^{n}=-\ln (1-x) /(1-x)$. $\S$ See Proposition 7.3.2 in [77].

$\dagger$ Not necessarily corresponding to modular forms as can be seen on (48), (49). 
where $R\left(x ; z_{1}, z_{2}, z_{3}\right)$ reads:

$$
\frac{z_{1} z_{2} z_{3}}{\left(1-x \cdot\left(1+z_{1}\right)\left(1+z_{2}\right)\left(1+z_{3}\right)\right)\left(z_{1} z_{2} z_{3}-x \cdot\left(1+z_{1}\right)\left(1+z_{2}\right)\left(1+z_{3}\right)\right)} .
$$

From this last result one deduces immediately that (37) is actually the diagonal of:

$$
\frac{1}{\left(1-z_{0} \cdot\left(1+z_{1}\right)\left(1+z_{2}\right)\left(1+z_{3}\right)\right) \cdot\left(1-z_{0} z_{1} z_{2} z_{3}\left(1+z_{1}\right)\left(1+z_{2}\right)\left(1+z_{3}\right)\right)} .
$$

Note that, as a consequence of a combinatorial identity due to Strehl and Schmidt $[85,86,87], \mathcal{S}(x)$ can also be written as

$$
\mathcal{S}(x)=\sum_{n=0}^{\infty} \sum_{k=0}^{n}\left(\begin{array}{l}
n \\
k
\end{array}\right)^{2}\left(\begin{array}{c}
2 k \\
n
\end{array}\right) \cdot x^{n}=\sum_{n=0}^{\infty} \sum_{k=[n / 2]}^{n}\left(\begin{array}{l}
n \\
k
\end{array}\right)^{2}\left(\begin{array}{c}
2 k \\
n
\end{array}\right) \cdot x^{n} .
$$

Calculations similar to (39) on this alternative binomial representation (40), enable to express (37) as the diagonal of an alternative rational function:

$$
\frac{1}{\left(1-z_{0} \cdot\left(1+z_{1}\right)\left(1+z_{2}\right)\left(1+z_{3}\right)^{2}\right) \cdot\left(1-z_{0} z_{1} z_{2} \cdot\left(1+z_{1}\right)\left(1+z_{2}\right)\right)} .
$$

We thus see that we can actually get explicitly, from straightforward calculations, the rational function (40) for the Calabi-Yau-like ODEs (occurring from differential geometry or enumerative combinatorics) when series with nested sums of binomials take place, and, more generally, for enumerative combinatorics problems (related or not to Calabi-Yau manifolds) where series with nested sums of binomials take place.

These effective calculations are actually algorithmic, and guarantee to obtain an explicit expression for the rational function (40). However the rational function is far from being unique, and worse, the number of variables, the rational function depends on, is far from being the smallest possible number. Finding the "minimal" rational function (whatever the meaning of "minimal" may be) is a very difficult problem. Appendix B provides a non-trivial illustration of this fact with explicit calculations on the well-known Apéry series and its rewriting due to Strehl and Schmidt [85, 86, 87]. We see in a crystal clear way in Appendix B that, when a given function is a diagonal of a rational function, the rational function is far from being unique, the "simplest" representation (minimal number of variables, lowest degree polynomials, ...) being hard to find. Similar computations $\dagger$ show that the generating function of sequence $\mathbf{B}$ and $\mathbf{E}$ in Zagier's list [83] are both diagonals of rationals function in four variables.

All these calculations can systematically be performed on any series defined by nested sums of product of binomials. We have performed such calculations on a large number of the series corresponding to the list of Almkvist et al [78], that are given by such nested sums of product of binomials.

\section{Comments and speculations}

\subsection{A theorem of [45]}

In [45] (page 61 Theorem 12, see also Proposition 7 in page 50 of [62]) it is proved that any power series with an integral representation and of maximal weight for the corresponding Picard-Fuchs linear differential equation is the diagonal of a rational function and, in particular, is globally bounded.

$\dagger$ These results are given in section (5.1) of [19]. 
The technical nature of the original papers is such that the result itself is difficult to find. This paragraph is devoted to explain, in down-to-earth terms, the somewhat esoteric expressions used in its wording, and to explain what it means on explicit examples. As the original proof is very obfuscated its principle is sketched in [19].

Disappointingly, when applied to a hypergeometric ${ }_{n+1} F_{n}$, this result becomes somewhat trivial. More precisely, the hypergeometric function is of maximal weight if and only if $b_{j}=1$ for all $j$ (there is only $n$ !'s in the denominator of coefficients). In that case it is obviously the Hadamard product of algebraic functions, therefore diagonal of a rational function:

$$
\begin{aligned}
{ }_{n} F_{n-1}\left(\left[\alpha_{1}, \alpha_{2}, \cdots, \alpha_{n}\right],[1,1, \cdots 1], x\right) \\
\quad=(1-x)^{-\alpha_{1}} \star(1-x)^{-\alpha_{2}} \cdots \star(1-x)^{-\alpha_{n}} .
\end{aligned}
$$

Therefore, we now have (at least) three sets of problems yielding diagonal of rational functions: the $n$-fold integrals of the form (34) with (35), the Picard-Fuchs linear ODEs with solution of maximal monodromy weight and, finally, the problems of enumerative combinatorics where nested sums of products of binomials take place.

Diagonal of rational functions, thus, occur in a quite large set of problems of theoretical physics. At first sight, one can see the frequent appearance of diagonals of rational functions in physics just as a mathematical curiousity $\dagger$, and be surprised that, for instance, so many series in physics are, modulo a prime, algebraic functions. Being diagonal of rational functions is not just as a mathematical curiousity: it corresponds (see next section) to G-operators, and their rational number exponents, and can be seen as a first step to modularity properties (see sections below) in some work-in-progress integrability.

\subsection{A conjecture of [45]}

The diagonal of a rational function is globally bounded (i.e. it has non zero radius of convergence and integer coefficients up to one rescaling) and $D$-finite (i.e. solution of a linear differential equation with polynomial coefficients) $\ddagger$.

The converse statement is the conjecture in [45] saying that any D-finite, globally bounded series is necessarily the diagonal of a rational function.

A remarkable result of Chudnovski's ([89] Chapter VIII) asserts that the minimal linear differential operator of a $G$-function (and in particular of a D-finite globally bounded series) is a $G$-operator (i.e. at least, a globally nilpotent operator) $[27,28,29]$. The conjecture in [45] amounts to saying something more: if the solution of this globally nilpotent linear differential operator is, not only a $G$-series, but a globally bounded series, then it is the diagonal of a rational function.

Conversely the solution, analytical at 0 , of a globally nilpotent linear differential operator is necessarily a $G$-function $[28,29]$. Moreover, a "classical" conjecture, with numerous avatars, claims that any $G$-function comes from geometry i.e. roughly speaking, it has an integral representation§.

To test the validity of the conjecture of [45] we look for counter-examples not contradicting classical conjectures. For instance, we search D-finite power series with

$\dagger$ In 1944 the occurrence of elliptic functions in Onsager's solution of the Ising model was also seen as a mathematical curiosity ...

$\ddagger$ The series expansion of the susceptibility of the isotropic 2-D Ising model can be recast into a series with integer coefficients (see $[12,18,26,88]$ ), but it cannot be the diagonal of rational functions since the full susceptibility is not a D-finite function [88].

$\S$ Bombieri-Dwork conjecture see for instance [29]. 
integer coefficients which are not algebraic but have an integral representation and are not of maximal weight for the corresponding Picard-Fuchs linear ODE.

As a first step let us limit ourselves to hypergeometric functions ${ }_{n+1} F_{n}$. The monodromy weight $W$ is exactly the number of 1 among the $b_{i}$.

When ${ }_{n+1} F_{n}$ is globally bounded and has no integer parameters $b_{i}(W=0)$, its minimal ODE has a $p$-curvature zero for almost all primes $p$. However, a Grothendieck conjecture, proved for ${ }_{3} F_{2}$ in [90], and generalised to ${ }_{n+1} F_{n}$ in [91], asserts that, under these circumstances, the hypergeometric function is algebraic. We display in Appendix $\mathrm{C}$ a set of ${ }_{n+1} F_{n}$ hypergeometric functions which yield, naturally, series with integer coefficients, many of them corresponding to such algebraic hypergeometric functions. Even if such examples are quite non-trivial, the purpose of our paper is to focus on transcendental (non algebraic) functions.

So we are looking for globally bounded hypergeometric functions satisfying $1 \leq W \leq n-1$. In general such hypergeometric functions are $G$-series but are very far from being globally bounded. The hypergeometric world extends largely outside the world of diagonal of rational functions.

Such an example in the first case $n=2, W=1$ was given in [45]:

$$
\begin{aligned}
& { }_{3} F_{2}\left(\left[\frac{1}{9}, \frac{4}{9}, \frac{5}{9}\right],\left[\frac{1}{3}, 1\right] ; 3^{6} x\right)=1+60 x+20475 x^{2}+9373650 x^{3} \\
& +4881796920 x^{4}+2734407111744 x^{5}+1605040007778900 x^{6}+\cdots
\end{aligned}
$$

The integer coefficients read with the rising factorial (or Pochhammer) symbol

$$
\frac{(1 / 9)_{n} \cdot(4 / 9)_{n} \cdot(5 / 9)_{n}}{(1 / 3)_{n} \cdot(1)_{n} \cdot n !} \cdot 3^{6 n}=\frac{\rho(n)}{\rho(0)},
$$

where:

$$
\rho(n)=\frac{\Gamma(1 / 9+n) \Gamma(4 / 9+n) \Gamma(5 / 9+n)}{\Gamma(1 / 3+n) \Gamma(1+n) \Gamma(1+n)} \cdot 3^{6 n} .
$$

Note that, at first sight, it is far from clear§ on (45), or on the simple recursion on the $\rho(n)$ coefficients (with the initial value $\rho(0)=1$ )

$$
\frac{\rho(n+1)}{\rho(n)}=3 \cdot \frac{(1+9 n)(4+9 n)(5+9 n)}{(1+3 n)(1+n)^{2}},
$$

to see that the $\rho(n)$ 's are actually integers. A sketch of the (quite arithmetic) proof that the $\rho(n)$ 's are actually integers, is given in Appendix D.

Because of the $1 / 3$ in the right (lower) parameters of (43), the hypergeometric function (43) is not an obvious Hadamard product of algebraic functions (and thus a diagonal of a rational function), and one can see that it is not an algebraic hypergeometric function either by calculating its $p$-curvature and finding that it is not zero [80] (see also [91, 92]). Proving that an algebraic function is the diagonal of a rational function and proving that a solution of maximal weight for a Picard-Fuchs equation is the diagonal of a rational function use two entirely distinct ways. The hope is to combine both techniques to conclude in the intermediate situation.

This example remained for twenty years, the only "blind spot" of the conjecture in [45]. We have recently found many other ${ }_{3} F_{2}$ examplesł, such that their series

$\S$ In contrast with cases where binomial (and thus integers) expressions take place.

$\ddagger{ }_{2} F_{1}$ cases are straightforward, and cannot provide counterexamples to conjecture in [45]. 
expansions have integer coefficients but are not obviously diagonals of rational functions. Some of these new hypergeometric examples $\dagger$ read for instance:

$$
\begin{aligned}
{ }_{3} F_{2}\left(\left[\frac{1}{9}, \frac{2}{9}, \frac{7}{9},\right],\left[\frac{2}{3}, 1\right], 3^{6} x\right)=1+21 x+5544 x^{2}+2194500 x^{3} & \\
+1032711750 x^{4}+535163031270 x^{5}+294927297193620 x^{6} & \\
+ & 169625328357359160 x^{7}+100668944872954458000 x^{8}+\cdots
\end{aligned}
$$

or:

$$
{ }_{3} F_{2}\left(\left[\frac{1}{7}, \frac{2}{7}, \frac{4}{7},\right],\left[\frac{1}{2}, 1\right], 7^{4} x\right), \quad{ }_{3} F_{2}\left(\left[\frac{1}{11}, \frac{2}{11}, \frac{6}{11},\right],\left[\frac{1}{2}, 1\right], 11^{4} x\right) .
$$

Unfortunately these hypergeometric examples are on the same "frustrating footing" as Christol's example (43): we are not able to show that one of them is actually a diagonal of a rational function, or, conversely, to show that one of them cannot be the diagonal of a rational function.

\section{Integrality versus modularity: learning by examples}

A large number of examples of integrality of series-solutions comes from modular forms. Let us just display two such modular forms associated with HeunG functions of the form $\operatorname{Heun} G(a, q, 1,1,1,1 ; x)$. Many more similar examples can be found in [19].

\subsection{First modular form example}

One can, for instance, rewrite the example (37) of subsection (4.2), namely $\operatorname{Heun} G(-1 / 8,1 / 4,1,1,1,1 ;-x)$, as a hypergeometric function with two rational pullbacks:

$$
\begin{aligned}
\text { HeunG }(-1 / 8,1 / 4,1,1,1,1 ;-x)=\sum_{n=0}^{\infty} \sum_{k=0}^{n}\left(\begin{array}{l}
n \\
k
\end{array}\right)^{3} x^{n} \\
=\left((1+4 x) \cdot\left(1+228 x+48 x^{2}+64 x^{3}\right)\right)^{-1 / 4} \\
\quad \times{ }_{2} F_{1}\left(\left[\frac{1}{12}, \frac{5}{12}\right],[1] ; \frac{1728 \cdot(1-8 x)^{6} \cdot(1+x)^{3} \cdot x}{\left(1+228 x+48 x^{2}+64 x^{3}\right)^{3} \cdot(1+4 x)^{3}}\right) \\
=\left((1-2 x) \cdot\left(1-6 x+228 x^{2}-8 x^{3}\right)\right)^{-1 / 4} \\
\quad \times{ }_{2} F_{1}\left(\left[\frac{1}{12}, \frac{5}{12}\right],[1] ; \frac{1728 \cdot(1-8 x)^{3} \cdot(1+x)^{6} \cdot x^{2}}{\left(1-6 x+228 x^{2}-8 x^{3}\right)^{3} \cdot(1-2 x)^{3}}\right) .
\end{aligned}
$$

The relation between the two pullbacks, that are related by the "Atkin" involution $x \leftrightarrow-1 / 8 / x$, gives the modular curve:

$$
\begin{gathered}
1953125 y^{3} z^{3}-187500 y^{2} z^{2} \cdot(y+z)+375 y z \cdot\left(16 z^{2}-4027 y z+16 y^{2}\right) \\
-64 \cdot(z+y) \cdot\left(y^{2}+z^{2}+1487 y z\right)+110592 \cdot y z=0 .
\end{gathered}
$$

$\dagger$ See also [19].

ๆ In previous papers [93, 30], with some abuse of language, we called such an involution an AtkinLehner involution. In fact this terminology is commonly used in the mathematical community for an involution $\tau \rightarrow-N / \tau$, on $\tau$, the ratio of periods, and not for our $x$-involution. This is why we switch to the wording "Atkin" involution. 
Series (48) is solution of the (exactly) self adjoint linear differential operator $\Omega$ where $\left(\theta=x \cdot D_{x}\right)$ :

$$
x \cdot \Omega=\theta^{2}-x \cdot\left(7 \theta^{2}+7 \theta+2\right)-8 x^{2} \cdot(\theta+1)^{2} .
$$

\subsection{Second modular form example}

The integrality of series-solutions can be quite non-trivial like the solution of the Apéry-like operator

$$
\begin{array}{lc}
\Omega= & x \cdot\left(1-11 x-x^{2}\right) \cdot D_{x}^{2}+\left(1-22 x-3 x^{2}\right) \cdot D_{x}-(x+3), \\
\text { or: } & x \cdot \Omega=\theta^{2}-x \cdot\left(11 \theta^{2}+11 \theta+3\right)-x^{2} \cdot(\theta+1)^{2},
\end{array}
$$

which can be written as a HeunG function. This (at first sight involved) HeunG function reads:

$$
\begin{aligned}
& \text { HeunG }\left(-\frac{123}{2}+\frac{55}{2} \cdot 5^{1 / 2},-\frac{33}{2}+\frac{15}{2} \cdot 5^{1 / 2}, 1,1,1,1 ;\left(\frac{11-5^{3 / 2}}{2}\right) \cdot x\right) \\
& =\sum_{n=0}^{\infty} \sum_{k=0}^{n}\left(\begin{array}{l}
n \\
k
\end{array}\right)^{2}\left(\begin{array}{c}
n+k \\
k
\end{array}\right) \cdot x^{n}=1+3 \cdot x+19 \cdot x^{2}+147 \cdot x^{3}+\cdots
\end{aligned}
$$

but actually corresponds to a modular form, which can be written in two different ways using two pullbacks:

$$
\begin{aligned}
\left(x^{4}+12 x^{3}+\right. & \left.14 x^{2}-12 x+1\right)^{-1 / 4} \\
\times & { }_{2} F_{1}\left(\left[\frac{1}{12}, \frac{5}{12}\right],[1] ; \frac{1728 \cdot x^{5} \cdot\left(1-11 x-x^{2}\right)}{\left(x^{4}+12 x^{3}+14 x^{2}-12 x+1\right)^{3}}\right) \\
=(1+ & \left.228 x+494 x^{2}-228 x^{3}+x^{4}\right)^{-1 / 4} \\
& \times{ }_{2} F_{1}\left(\left[\frac{1}{12}, \frac{5}{12}\right],[1] ; \frac{1728 \cdot x \cdot\left(1-11 x-x^{2}\right)^{5}}{\left(1+228 x+494 x^{2}-228 x^{3}+x^{4}\right)^{3}}\right) .
\end{aligned}
$$

Modular form examples of series with integer coefficients displayed in Appendix A, correspond to lattice Green functions [48]. Therefore, they have $n$ fold integral representations $\dagger$, and, after section (3.4), can be seen to be diagonals of rational functions.

\section{Integrality versus modularity}

\subsection{Diffeomorphisms of unity pullbacks}

Let us consider a first simple example of a hypergeometric function which is solution of a Calabi-Yau ODE, and which occurred, at least two times in the study of the Ising susceptibility $n$-fold integrals $[30,31] \chi^{(n)}$ and $\chi_{d}^{(n)}$, namely ${ }_{4} F_{3}([1 / 2,1 / 2,1 / 2,1 / 2],[1,1,1] ; 256 x)$, where we perform a (diffeomorphism of unity) pullback:

$$
\begin{aligned}
{ }_{4} F_{3}\left(\left[\frac{1}{2},\right.\right. & \left.\left.\frac{1}{2}, \frac{1}{2}, \frac{1}{2}\right],[1,1,1] ; \frac{256 x}{1+c_{1} x+c_{2} x^{2}+\cdots}\right)=1+16 \cdot x \\
& +16 \cdot\left(81-c_{1}\right) \cdot x^{2}+16 \cdot\left(10000+c_{1}^{2}-c_{2}-162 c_{1}\right) \cdot x^{3}+\cdots
\end{aligned}
$$

$\dagger$ In contrast the modular form examples displayed in Appendix $\mathrm{H}$ of [19] correspond to differential geometry examples discovered by Golyshev and Stienstra [94], where no $n$-fold integral representation is available at first sight. 
If the pull-back in (53) is such that the coefficients $c_{n}$, at its denominator, are integers, one finds that the series expansion is actually a series with integer coefficients, for every such pullback (i.e. for every integer coefficients $c_{n}$ ). Furthermore, a straightforward calculation of the corresponding nome $q(x)$ and its compositional inverse (mirror map) $x(q)$, also yields series with integer coefficients:

$$
\begin{aligned}
& q(x)=x+\left(64-c_{1}\right) \cdot x^{2}+\left(c_{1}^{2}+7072-c_{2}-128 c_{1}\right) \cdot x^{3}+\cdots, \\
& x(q)=q+\left(c_{1}-64\right) \cdot q^{2}+\left(c_{1}^{2}+1120+c_{2}-128 c_{1}\right) \cdot q^{3}+\cdots,
\end{aligned}
$$

when its Yukawa coupling [30], seen as a function of the nome $q, K(q)$ is also a series with integer coefficients and is independent of the pullback:

$$
K(q)=1+32 \cdot q+4896 \cdot q^{2}+702464 \cdot q^{3}+\cdots
$$

This independence of the Yukawa coupling with regards to pullbacks, is a known property, and has been proven in [75], for any pullback of the diffeomorphism of unity form $p(x)=x+\cdots$

Seeking for Calabi-Yau ODEs, Almkvist et al. have obtained [78] a quite large list of fourth order ODEs, which are MUM by definition and have, by construction, the integrality for the solution-series analytic at $x=0$. Looking at the Yukawa coupling of these ODEs is a way to define equivalence classes up to pullbacks of ODEs sharing the same Yukawa coupling. This "wraps in the same bag" all the linear ODEs that are the same up to pullbacks. Let us recall how difficult it is to see if a given Calabi-Yau ODE has, up to operator equivalence, and up to pullback, a hypergeometric function solution [30,31], because finding the pullback is extremely difficult [30,31]. We may have, for the Ising model, some ${ }_{n+1} F_{n}$ hypergeometric function prejudice [30,31]: it is, then, important to have an invariant that is independent of this pullback that we cannot find most of the time.

Finally, let us remark that the Yukawa coupling is not preserved by the operator equivalence. Two linear differential operators, that are homomorphic, do not necessarily have the same Yukawa coupling (see Appendix E).

\subsection{Yukawa couplings in terms of determinants}

Another way to understand this fundamental pullback invariance, amounts to rewriting the Yukawa coupling [75, 95], not from the definition usually given in the literature (second derivative with respect to the ratio of periods), but in terms of determinants of solutions (Wronskians, ...) that naturally present nice covariance properties with respect to pullback transformations (see Appendix E).

We have the alternative definition for the Yukawa coupling given in Appendix E:

$$
K(q)=\left(q \cdot \frac{d}{d q}\right)^{2}\left(\frac{y_{2}}{y_{0}}\right)=\frac{W_{1}^{3} \cdot W_{3}}{W_{2}^{3}},
$$

where the determinantal variables $W_{m}$ 's are determinants built from the four solutions of the MUM differential operator. This alternative definition, in terms of these $W_{m}$ 's, enables to understand the remarkable invariance of the Yukawa coupling by pullback transformations [31]. These determinantal variables $W_{m}$ quite naturally, and canonically, yield to introduce another "Yukawa coupling" (which, in fact, corresponds to the Yukawa coupling of the adjoint operator (see E.12)). This "adjoint Yukawa coupling" is also invariant by pullbacks. It has, for the previous example, the following series expansion with integer coefficients:

$$
K^{\star}(q)=1+32 \cdot q+4896 \cdot q^{2}+702464 \cdot q^{3}+\cdots
$$


which actually identifies with (56). The equality of the Yukawa coupling for this orderfour operator, and for its (formal) adjoint operator, is a straightforward consequence of the fact that the order-four operator annihilating ${ }_{4} F_{3}\left(\left[\frac{1}{2}, \frac{1}{2}, \frac{1}{2}, \frac{1}{2}\right],[1,1,1] ; 256 x\right)$ is exactly self-adjoint, and, more generally, of the fact that the order-four operator, annihilating (53), is conjugated to its adjoint by a simple function.

\subsection{Modularity}

This example, with its corresponding relations (53), (54), (56), (58) may suggest a quite wrong prejudice that the integrality of the solution of an order-four linear differential operator automatically yields to the integrality of the nome, mirror map and Yukawa coupling, that we will call, for short, "modularity". This is far from being the case, as can be seen, for instance, in the following interesting example, where the nome and Yukawa coupling $K(q)$ do not correspond to globally bounded series, when the ${ }_{4} F_{3}$ solution of the order-four operator as well as the Yukawa coupling seen as a function of $x, K(x)$, are, actually, both series with integer coefficients.

Let us consider the following ${ }_{4} F_{3}$ hypergeometric function which is clearly a Hadamard product of algebraic functions and, thus, the diagonal of a rational function:

$$
\begin{aligned}
{ }_{4} F_{3}\left(\left[\frac{1}{2},\right.\right. & \left.\left.\frac{1}{3}, \frac{1}{4}, \frac{3}{4}\right],[1,1,1] ; x\right) \\
& =(1-x)^{-1 / 3} \star(1-x)^{-1 / 2} \star(1-x)^{-1 / 4} \star(1-x)^{-3 / 4} \\
& =\operatorname{Diag}\left(\left(1-z_{1}\right)^{-1 / 3}\left(1-z_{2}\right)^{-1 / 2}\left(1-z_{3}\right)^{-1 / 4}\left(1-z_{4}\right)^{-3 / 4}\right),
\end{aligned}
$$

It is therefore globally bounded:

$$
\begin{gathered}
{ }_{4} F_{3}\left(\left[\frac{1}{2}, \frac{1}{3}, \frac{1}{4}, \frac{3}{4}\right],[1,1,1] ; 2304 x\right)=1+72 x+45360 x^{2}+46569600 x^{3} \\
+59594535000 x^{4}+86482063571904 x^{5}+\cdots
\end{gathered}
$$

Its Yukawa coupling, seen as a function of $x$, is actually a series with integer coefficients in $x$ :

$$
\begin{aligned}
K(x)=1 & +480 x+872496 x^{2}+1728211968 x^{3}+3566216754432 x^{4} \\
& +7536580798814208 x^{5}+16177041308360579328 x^{6}+\cdots
\end{aligned}
$$

However, do note that the series, in term of the nome, is not globally bounded:

$$
\begin{aligned}
K(q)=1 & +480 q+653616 q^{2}+942915456 q^{3}+1408019875200 q^{4}+\cdots \\
& +571436303929319146711343817202689132288 \frac{q^{12}}{11}+\cdots
\end{aligned}
$$

In fact, the nome $q(x)$, and the mirror map $x(q)$, are also not globally bounded. Note that in this example, the non integrality appears at order twelve (for $x(q), q(x)$ and $K(q)$ ). If the prime 11 in the denominator in (61) was the only one, one could recast the series into a series with integer coefficients introducing another rescaling $2304 x \rightarrow 11 \times 2304 x$. But, in fact, we do see the appearance of an infinite number of other primes at higher orders denominators in $x(q), q(x)$ and $K(q)$.

We do not have modularity because we do not have (up to rescaling) the nome integrality: the nome series is not globally bounded. 


\subsection{Order-two differential operators $\omega_{n}$ associated with modular forms}

After Maier [96] let us underline that modular forms can be written as hypergeometric functions with two different pullbacks, and, consequently, one can associate order-two differential operators to these modular forms.

Let us consider the two order-two operators

$$
\begin{aligned}
& \omega_{2}=D_{x}^{2}+\frac{(96 x+1)}{(64 x+1) \cdot x} \cdot D_{x}+\frac{4}{(64 x+1) x}, \\
& \omega_{3}=D_{x}^{2}+\frac{(45 x+1)}{(27 x+1) \cdot x} \cdot D_{x}+\frac{3}{(27 x+1) x}
\end{aligned}
$$

which are associated with two modular forms corresponding, on their associated nomes $q$, to the transformations $q \rightarrow q^{2}$ and $q \rightarrow q^{3}$ respectively (multiplication of $\tau$, the ratio of their periods by 2 and 3 ), as can be seen on their respective solutions:

$$
\begin{aligned}
{ }_{2} F_{1}\left(\left[\frac{1}{4}, \frac{1}{4}\right],[1] ;-64 x\right)=(1+256 x)^{-1 / 4} \cdot{ }_{2} F_{1}\left(\left[\frac{1}{12}, \frac{5}{12}\right],[1] ; \frac{1728 x}{(1+256 x)^{3}}\right) \\
=(1+16 x)^{-1 / 4} \cdot{ }_{2} F_{1}\left(\left[\frac{1}{12}, \frac{5}{12}\right],[1] ; \frac{1728 x^{2}}{(1+16 x)^{3}}\right) \\
=1-4 x+100 x^{2}-3600 x^{3}+152100 x^{4}-7033104 x^{5}+344622096 x^{6} \\
\quad-17582760000 x^{7}+924193822500 x^{8}-49701090010000 x^{9}+\cdots
\end{aligned}
$$

and:

$$
\begin{aligned}
& \left((1+27 x)(1+243 x)^{3}\right)^{-1 / 12} \cdot{ }_{2} F_{1}\left(\left[\frac{1}{12}, \frac{5}{12}\right],[1] ; \frac{1728 x}{(1+243 x)^{3}(1+27 x)}\right) \\
& =\left((1+27 x)(1+3 x)^{3}\right)^{-1 / 12} \cdot{ }_{2} F_{1}\left(\left[\frac{1}{12}, \frac{5}{12}\right],[1] ; \frac{1728 x^{3}}{(1+3 x)^{3}(1+27 x)}\right) \quad(65) \\
& ={ }_{2} F_{1}\left(\left[\frac{1}{3}, \frac{1}{3}\right],[1],-27 x\right)=1-3 x+36 x^{2}-588 x^{3}+11025 x^{4}-223587 x^{5} \\
& \quad+4769856 x^{6}-105423552 x^{7}+2391796836 x^{8}-55365667500 x^{9}+\cdots
\end{aligned}
$$

The relation between the two Hauptmodul pullbacks in (64)

$$
u=\frac{1728 x}{(1+256 x)^{3}}, \quad v=\frac{1728 x^{2}}{(1+16 x)^{3}}=u\left(\frac{1}{2^{12} x}\right),
$$

corresponds to the (genus-zero) fundamental modular curve:

$$
\begin{gathered}
5^{9} \cdot u^{3} v^{3}-12 \cdot 5^{6} \cdot u^{2} v^{2} \cdot(u+v)+375 u v \cdot\left(16 u^{2}+16 v^{2}-4027 u v\right) \\
-64(u+v) \cdot\left(v^{2}+1487 u v+u^{2}\right)+2^{12} 3^{3} \cdot u v=0 .
\end{gathered}
$$

The relation between the two Hauptmodul pullbacks in (65)

$$
u=\frac{1728 x}{(1+243 x)^{3}(1+27 x)}, \quad v=\frac{1728 x^{3}}{(1+3 x)^{3}(1+27 x)}=u\left(\frac{1}{3^{6} x}\right),
$$

corresponds to the (genus-zero) modular curve:

$$
\begin{gathered}
2^{27} 5^{9} \cdot u^{3} v^{3} \cdot(u+v)+2^{18} 5^{6} u^{2} v^{2} \cdot\left(27 v^{2}+27 u^{2}-45946 u v\right) \\
+2^{9} 3^{5} 5^{3} u v \cdot(u+v) \cdot\left(v^{2}+241433 u v+u^{2}\right) \\
+729\left(u^{4}+v^{4}\right)-39628 \cdot 3^{9} \cdot\left(u^{2}+v^{2}\right) \cdot u v+15974803 \cdot 2 \cdot 3^{10} \cdot u^{2} v^{2} \\
+31 \cdot 2^{9} 3^{11} u v \cdot(u+v)-2^{12} 3^{12} u v=0 .
\end{gathered}
$$


Similarly, one can consider the order-two operators $\omega_{n}$ associated with other modular forms corresponding to $\tau \rightarrow n \cdot \tau$. The $\omega_{n}$ 's can be simply deduced from Maier [96], for modular forms corresponding to genus-zero curves i.e. for $n=2,3$, $4,5,6,7,8,9,10,12,13,16,18,25$. Since the solutions can be written as ${ }_{2} F_{1}$ hypergeometric up to rational pullbacks, these genus-zero $\omega_{n}$ 's are obviously ordertwo operators. After a simple rescaling, the solutions analytic at $x=0$, can be rewritten as series with integer coefficients.

One can also consider the other $\omega_{n}$ 's corresponding to higher-genus modular curves. In these cases, one does not have a rational parametrisation like (66) or (68), but one still has an identity of the same hypergeometric function with two different pullbacks, these two pullbacks being algebraic functions and not rational functions (see (66) or (68)). These algebraic functions correspond to the so-called modular polynomials [19].

For instance for $\tau \rightarrow 11 \cdot \tau$, one has a genus-one modular curve, the modular polynomial reads:

$$
\begin{aligned}
& P_{11}^{*}(x, H)=\left(1+228 x+486 x^{2}-540 x^{3}+225 x^{4}\right)^{3} \cdot H^{2} \\
& -1728 \cdot \mathcal{Q}_{1}(x) \cdot x \cdot H \quad+1728^{2} x^{12}, \quad \text { with: } \\
& \mathcal{Q}_{1}(x)=1-55 x+1188 x^{2}-12716 x^{3}+69630 x^{4}-177408 x^{5}+133056 x^{6} \\
& +132066 x^{7}-187407 x^{8}+40095 x^{9}+24300 x^{10}-6750 x^{11} .
\end{aligned}
$$

One has the identity

$$
{ }_{2} F_{1}\left(\left[\frac{1}{12}, \frac{5}{12}\right],[1] ; H_{1}\right)=A(x) \cdot{ }_{2} F_{1}\left(\left[\frac{1}{12}, \frac{5}{12}\right],[1] ; H_{2}\right),
$$

where the two Hauptmoduls $H_{1}$ and $H_{2}$ are the two solutions of $P_{11}^{*}(x, H)=0$, and where $A(x)$ is the algebraic function such that

$$
\frac{A(x)^{4}}{11^{2}}+\frac{11^{2}}{A(x)^{4}}=\frac{2}{11^{2}} \cdot \frac{7321-87612 x+73206 x^{2}+21060 x^{3}-23175 x^{4}}{1+228 x+486 x^{2}-540 x^{3}+225 x^{4}},
$$

this last relation between $A(x)^{4}$ and $x$ corresponding to a genus-one curve with the same $j$-invariant as the genus-one curve $P_{11}^{*}(x, H)=0$, namely $j=-496^{3} / 11^{5}$. The two hypergeometric functions in (71) are actually series with integer coefficients:

$$
\begin{gathered}
{ }_{2} F_{1}\left([1 / 12,5 / 12],[1] ; H_{1}\right)=1+60 x-4560 x^{2}+614400 x^{3}-95660400 x^{4} \\
+16231863060 x^{5}-2905028387700 x^{6}+\cdots \\
{ }_{2} F_{1}\left([1 / 12,5 / 12],[1] ; H_{2}\right)=1+60 x^{11}+3300 x^{12}+110220 x^{13}+2904660 x^{14} \\
+66599940 x^{15}+1394683620 x^{16}+27425371380 x^{17}+\cdots
\end{gathered}
$$

More details are given for this $\tau \rightarrow 11 \cdot \tau$ case in Appendix I of [19]. Note that although ${ }_{2} F_{1}\left([1 / 12,5 / 12],[1] ; H_{1}\right)$ and ${ }_{2} F_{1}\left([1 / 12,5 / 12]\right.$, [1]; $\left.H_{2}\right)$ are solutions of the same order-four operator [19], one can find an appropriate algebraic function $\mathcal{A}(x)$, such that $\mathcal{A}(x) \cdot{ }_{2} F_{1}\left([1 / 12,5 / 12],[1], H_{1}\right)$ is solution of an order-two operator $\omega_{11}$ (see [19] for more details).

The other $\omega_{n}$ 's, corresponding to higher genus modular curves [97], are actually also order-two operators. The explicit expressions of $\omega_{n}$ 's for the elliptic values $n=17,19$, and the hyperelliptic values [97] $n=23,29,31,41,47,59,71$ are given in [19]. The genus of the associated modular curves [97], is respectively [19] genusone for $\tilde{\omega}_{17}(x), \tilde{\omega}_{19}(x)$, genus-two for $\tilde{\omega}_{23}(x), \tilde{\omega}_{29}(x)$, $\tilde{\omega}_{31}(x)$, genus-three for $\tilde{\omega}_{41}(x)$, genus-four for $\tilde{\omega}_{47}(x)$, genus-five for $\tilde{\omega}_{59}(x)$, and genus-six for $\tilde{\omega}_{71}(x)$. 


\subsection{Hadamard products of $\omega_{n}$ 's}

The two operators $\omega_{2}$ and $\omega_{3}$ have a "modularity" property: their series expansions analytic at $x=0,(64)$ and (65), as well as the corresponding nomes, mirror maps are series with integer coefficients. The Hadamard product is a quite natural operation to introduce because it preserves the global nilpotence of the operators, it preserves the integrality of series-solutions, and it is a natural operation to introduce when seeking for diagonals of rational functions $\uparrow$. Let us perform the Hadamard product of these two operators. With some abuse of language [31], the Hadamard product of the two order-two operators (62) and (63)

$$
\begin{aligned}
H_{2,3}=\omega_{2} \star \omega_{3} & =D_{x}^{4}+6 \frac{(2064 x-1)}{(1728 x-1) \cdot x} \cdot D_{x}^{3}+\frac{(19020 x-7)}{(1728 x-1) \cdot x^{2}} \cdot D_{x}^{2} \\
& +\frac{(4788 x-1)}{(1728 x-1) \cdot x^{3}} \cdot D_{x}+\frac{12}{(1728 x-1) \cdot x^{3}}
\end{aligned}
$$

is defined as the (minimal order) linear differential operator having, as a solution, the Hadamard product of the solution-series (64) and (65), which is, by construction, a series with integer coefficients. This series is, of course, nothing but the expansion of the hypergeometric function:

$$
\begin{aligned}
{ }_{4} F_{3}\left(\left[\frac{1}{4}, \frac{1}{4}, \frac{1}{3}, \frac{1}{3}\right],[1,1,1] ; 1728 x\right) \\
\quad={ }_{2} F_{1}\left(\left[\frac{1}{4}, \frac{1}{4}\right],[1] ;-64 x\right) \star{ }_{2} F_{1}\left(\left[\frac{1}{3}, \frac{1}{3}\right],[1] ;-27 x\right) .
\end{aligned}
$$

In a similar way one can consider (see [19]) $H_{2,2}=\omega_{2} \star \omega_{2}$ (resp. $H_{3,3}=$ $\left.\omega_{3} \star \omega_{3}\right)$ the Hadamard product of the order-two operator (62) (resp. (63)) with itself (Hadamard square). These two operators have respectively the hypergeometric solutions

$$
{ }_{4} F_{3}\left(\left[\frac{1}{4}, \frac{1}{4}, \frac{1}{4}, \frac{1}{4}\right],[1,1,1] ; 4096 x\right), \quad{ }_{4} F_{3}\left(\left[\frac{1}{3}, \frac{1}{3}, \frac{1}{3}, \frac{1}{3}\right],[1,1,1] ; 729 x\right),
$$

corresponding to series expansions with integer coefficients. These operators $H_{2,2}$, $H_{3,3}$ are MUM operators. We can, therefore, define, without any ambiguity, the nome (and mirror map) and Yukawa coupling of this order-four operator [31]. One finds out that the nome $\dagger$, and the mirror map (and the Yukawa coupling as a function of the $x$ variable), are not globally bounded: they cannot be reduced, by one rescaling, to series with integer coefficients.

The three linear differential operators $H_{2,3}, H_{2,2}$ and $H_{3,3}$, are MUM and of order four, however, they are not of the Calabi-Yau type.

\subsection{Hadamard products versus Calabi-Yau ODEs}

The occurrence of Calabi-Yau type operators, that we could imagine, at first sight, to be extremely rare, is in fact quite frequent among such Hadamard products, as can be seen with other values of $n$ and $m$. For instance, one can introduce $\ddagger$ $H_{4,4}=\omega_{4} \star \omega_{4}$, the Hadamard square of $\omega_{4}$, which is an irreducible order-four

I And, consequently, has been heavily used to build Calabi-Yau-like ODEs (see Almkvist et al. [75]). $\dagger$ The nome of the Hadamard product of two operators has no simple relation with the nome of these two linear differential operators.

$\ddagger$ To get the Hadamard product of two linear differential operators use, for instance, Maple's command gfun[hadamardproduct]. 
linear differential operator, and has the hypergeometric solution already encountered for some $n$-fold integrals of the decomposition of the full magnetic susceptibility of the Ising model [30, 31] (see also subsections (7.1) and (7.2)):

$$
\begin{aligned}
{ }_{4} F_{3}\left(\left[\frac{1}{2}, \frac{1}{2}, \frac{1}{2}, \frac{1}{2}\right],\right. & {[1,1,1] ; 256 x) } \\
& ={ }_{2} F_{1}\left(\left[\frac{1}{2}, \frac{1}{2}\right],[1] ;-16 x\right) \star{ }_{2} F_{1}\left(\left[\frac{1}{2}, \frac{1}{2}\right],[1] ;-16 x\right) .
\end{aligned}
$$

The associated operator having (77) as a solution, obeys the "Calabi-Yau condition" that its exterior square is of order five.

Let us give in a table the orders (which go from 4 to 20) of the various $H_{m, n}=H_{n, m}$ Hadamard products of the order-two operators associated with the (genus-zero) modular forms operators $\omega_{n}$ and $\omega_{m}$ :

\begin{tabular}{|l|l|l|l|l|l|l|l|l|l|l|l|l|l|l|}
\hline $\mathrm{n} \backslash \mathrm{m}$ & 2 & 3 & 4 & 5 & 6 & 7 & 8 & 9 & 10 & 12 & 13 & 16 & 18 & 25 \\
\hline 2 & 4 & 4 & 4 & 6 & 4 & 6 & 4 & 4 & 10 & 8 & 10 & 8 & 12 & 14 \\
\hline 3 & & 4 & 4 & 6 & 4 & 6 & 4 & 4 & 10 & 8 & 10 & 8 & 12 & 14 \\
\hline 4 & & & $4 *$ & 6 & $4 *$ & 6 & $4 *$ & $4 *$ & 10 & 8 & 10 & 8 & 12 & 14 \\
\hline 5 & & & & 6 & 6 & 8 & 6 & 6 & 12 & 10 & 12 & 10 & 14 & 16 \\
\hline 6 & & & & & $4 *$ & 6 & $4 *$ & $4 *$ & 10 & 8 & 10 & 8 & 12 & 14 \\
\hline 7 & & & & & & 6 & 6 & 6 & 12 & 10 & 12 & 10 & 14 & 16 \\
\hline 8 & & & & & & & $4 *$ & $4 *$ & 10 & 8 & 10 & 8 & 12 & 14 \\
\hline 9 & & & & & & & & $4 *$ & 10 & 8 & 10 & 8 & 12 & 14 \\
\hline 10 & & & & & & & & & 10 & 14 & 16 & 14 & 18 & 20 \\
\hline 12 & & & & & & & & & & 8 & 14 & 12 & 16 & 18 \\
\hline 13 & & & & & & & & & & & 10 & 14 & 18 & 20 \\
\hline 16 & & & & & & & & & & & & 8 & 16 & 18 \\
\hline 18 & & & & & & & & & & & & & 12 & 20 \\
\hline 25 & & & & & & & & & & & & & & 14 \\
\hline
\end{tabular}

where the star $*$ denotes Calabi-Yau ODEs $\sharp$.

The following operators are of order four: $H_{2,2}, H_{2,3}, H_{2,4}, H_{2,6}, H_{2,8}, H_{2,9}$, $H_{3,3}, H_{3,4}, H_{3,6}, H_{3,8}, H_{3,9}, \ldots$ Their exterior squares, which are of order six, do not have rational solutions $\dagger$.

The order-four operators $H_{3,3}, H_{3,4}$, are all MUM operators $\uparrow$, but, similarly to the situation encountered with $H_{2,2}$, their nome, mirror map and Yukawa couplings are not globally bounded.

The following operators are of order six: $H_{2,5}, H_{2,7}, H_{3,5}, H_{3,7}, H_{4,5}, H_{4,7}$, $H_{5,5}, H_{5,6}, H_{5,8}, H_{5,9}, H_{6,7}, H_{7,7}, H_{7,8}, H_{7,9}, \ldots$ Their exterior square, which are of order fifteen, do not have rational solutions (and cannot be homomorphic to higher order Calabi-Yau linear ODEs).

Remarkably the following ten order-four operators $H_{4,4}, H_{4,6}, H_{4,8}, H_{4,9}, H_{6,6}$, $H_{6,8}, H_{6,9}, H_{8,8}, H_{8,9}, H_{9,9}$ (with a star in the previous table) are all MUM, and are

$\sharp$ Recall that Calabi-Yau ODEs are defined by a list of constraints [75], the most important ones being, besides being MUM, that their exterior square are of order five. There are more exotic conditions like the cyclotomic condition on the monodromy at $\infty$, see Proposition 3 in [78].

$\dagger$ They cannot be homomorphic to Calabi-Yau ODEs.

I Note that the Hadamard product of two MUM ODEs is not necessarily a MUM ODE: the order-six operator $H_{3,7}$ is not MUM. 
such that their exterior squares are of order five§: they are Calabi-Yau ODEs. Actually the nome, mirror map and Yukawa coupling series are series with integer coefficients for all these order-four Calabi-Yau operators. Their Yukawa coupling and their adjoint Yukawa coupling identify. The Yukawa coupling series of these Calabi-Yau operators are respectively, for $H_{4,4}$

$$
\begin{aligned}
K(q) & =K^{\star}(q)=1+32 \cdot q+4896 \cdot q^{2}+702464 \cdot q^{3}+102820640 \cdot q^{4} \\
& +15296748032 \cdot q^{5}+2302235670528 \cdot q^{6}+\cdots
\end{aligned}
$$

which is \#3 in Almkvist et al. large tables of Calabi-Yau ODEs [78], and is the well-known one for ${ }_{4} F_{3}([1 / 2,1 / 2,1 / 2,1 / 2],[1,1,1] ; 256 x)$, and for $H_{4,6}$

$$
\begin{aligned}
K(q)= & K^{\star}(q)=1+20 \cdot q+36 \cdot q^{2}+15176 \cdot q^{3}+486564 \cdot q^{4} \\
& +21684020 \cdot q^{5}+1209684456 \cdot q^{6}+\cdots
\end{aligned}
$$

which is \#137 in tables [78].

We give, in [19], the expansion of the Yukawa coupling for a set of other $H_{m, n}$ such that their exterior squares are order five (not six as one could expect for a generic irreducible order-four operator), that actually are Calabi-Yau operators. Actually operator $H_{4,8}$ is \#36 in Almkvist et al. large tables of Calabi-Yau ODEs [78]. Operators $H_{4,9}, H_{6,6}, H_{6,8}$ and $H_{6,9}$ are respectively [78] \#133, \#144, \#176 and \#178. Furthermore, operators $H_{8,8}, H_{8,9}$ and $H_{9,9}$ are respectively [78] \#107, \#163 and \#165.

It will be shown, in a forthcoming publication, that the occurrence of an order five for the exterior power (the "Calabi-Yau condition") means that these operators are necessarily conjugated (by an algebraic function) to their adjoints. Thus, the "adjoint Yukawa coupling" $K^{\star}(q)$ is necessarily equal to the Yukawa coupling $K(q)$ for these operators.

On the other hand, the ten linear differential operators denoted by a star $*$ in the previous table all share the same property: they have, as a solution, the Hadamard product of two HeunG functions solutions of the form $\operatorname{Heun} G(a, q, 1,1,1,1 ; x)$. Note, however, that this HeunG-viewpoint of the most interesting $H_{m, n}$ 's does not really help. Even inside this restricted set of HeunG functions solutions of the form $\operatorname{Heun} G(a, q, 1,1,1,1 ; x)$ it is hard to find exhaustively the values of the parameter $a$ and of the accessory parameter $q$, such the series $\operatorname{Heun} G(a, q, 1,1,1,1 ; x)$ is globally bounded, or, just, such that the order-two operator, having $\operatorname{Heun} G(a, q, 1,1,1,1 ; x)$ as a solution, is globally nilpotent [27].

Many $H_{m, n}$ are not MUM, for instance the order-eight operator $H_{12,12}$, or the order-six operator $H_{3,7}$, are not MUM. Concerning $H_{3,7}$, and as far as its six solutions are concerned, they are structured "like" the four solutions of an order-four MUM operator, together with the two solutions of another order-two MUM operator, but the order-six operator $H_{3,7}$ is not a direct-sum of an order-four and order-two operator. We have two solutions analytical at $x=0$ (with no logarithmic terms), and two solutions involving $\ln (x)$. A linear combination of these two solutions analytical at $x=0$ is, by construction a series with integer coefficients (the Hadamard product of the two series with integer coefficients which are the initial ingredients in this calculation), when the other linear combinations are not globally bounded.

$\S$ They are conjugated to their (formal) adjoint by a function. 


\section{Calabi-Yau Modularity}

The previous examples correspond to a "modularity" inherited from elliptic curves, more precisely Hadamard products of modular forms. Let us consider, here, two Calabi-Yau examples that do not seem to be reducibleł to ${ }_{4} F_{3}$ hypergeometric functions.

A first order-four Calabi-Yau operator, found by Batyrev and van Straten [77], which is self-adjoint and also corresponds to Hadamard products of simple hypergeometric functions (see (F.2)), is given in Appendix F. All the associated series (solution (F.2), nome, Yukawa coupling) are series expansion with integer coefficients. We do not have a representation of the solution (F.2), as an $n$-fold integral of the form (34). However, since (F.2) can be expressed as a sum of products of binomials (see (F.3)), we can conclude, again, that (F.2) is actually a diagonal of a rational function.

\subsection{A Batyrev and van Straten Calabi-Yau ODE [77]}

A second example of order-four operator, corresponding to Calabi-Yau 3-folds in $P_{1} \times P_{1} \times P_{1} \times P_{1}$, has been found by Batyrev and van Straten [77] (see† page 34):

$$
\begin{aligned}
B_{2}=\theta^{4}-4 x & \cdot\left(5 \theta^{2}+5 \theta+2\right) \cdot(2 \theta+1)^{2} \\
+ & 64 x^{2} \cdot(2 \theta+3) \cdot(2 \theta+1) \cdot(2 \theta+2)^{2} .
\end{aligned}
$$

It corresponds to the series-solution with coefficients:

$$
\left(\begin{array}{c}
2 n \\
n
\end{array}\right) \cdot \sum_{k=0}^{n}\left(\begin{array}{l}
n \\
k
\end{array}\right)^{2} \cdot\left(\begin{array}{c}
2 k \\
k
\end{array}\right) \cdot\left(\begin{array}{c}
2 n-2 k \\
n-k
\end{array}\right) .
$$

Its Wronskian $W_{4}$ is a rational function such that:

$$
x^{3} \cdot W_{4}^{1 / 2}=\frac{1}{(1-64 x)(1-16 x)} .
$$

This operator is also a Calabi-Yau operator: it is MUM, and it is such that its exterior square is order five. This order five property is a consequence of $B_{2}$ being conjugated to its adjoint: $B_{2} \cdot x=x \cdot \operatorname{adjoint}\left(B_{2}\right)$.

The series-solution of (80) can be written as an Hadamard product

$$
\begin{aligned}
\mathcal{S} & =(1-4 x)^{-1 / 2} \star \operatorname{HeunG}(4,1 / 2,1 / 2,1 / 2,1,1 / 2 ; 16 x)^{2} \\
& =1+8 x+168 x^{2}+5120 x^{3}+190120 x^{4}+7939008 x^{5}+357713664 x^{6}+\cdots,
\end{aligned}
$$

the modular form character of $\operatorname{Heun} G(4,1 / 2,1 / 2,1 / 2,1,1 / 2 ; 16 x)$ being illustrated with identities (A.4) in Appendix A of [19]. Its nome reads:

$$
\begin{aligned}
q & =x+20 x^{2}+578 x^{3}+20504 x^{4}+826239 x^{5}+36224028 x^{6}+1684499774 x^{7} \\
& +81788693064 x^{8}+4104050140803 x^{9}+211343780948764 x^{10}+\cdots
\end{aligned}
$$

The mirror map of (80) reads:

$$
\begin{aligned}
& x(q)=q-20 q^{2}+222 q^{3}-2704 q^{4}+21293 q^{5}-307224 q^{6}+80402 q^{7} \\
& -67101504 q^{8}-1187407098 q^{9}-37993761412 q^{10}+\cdots
\end{aligned}
$$

$\ddagger$ The possibility that a solution of an order-four operator, non-trivially equivalent to these CalabiYau operators [77], could be written as a ${ }_{4} F_{3}$ hypergeometric function, up to an involved algebraic pullback, is not totally excluded. However it is extremely difficult to rule out such highly non-trivial hypergeometric scenario.

$\dagger$ There is a small misprint in [77] page 34: $(2 \theta+1)$ must be replaced by $(2 \theta+1)^{2}$ in the $4 x$ term. 
The Yukawa coupling of (80) reads:

$$
\begin{gathered}
K(q)=K^{\star}(q)=1+4 q+164 q^{2}+5800 q^{3}+196772 q^{4}+6564004 q^{5} \\
+222025448 q^{6}+7574684408 q^{7}+259866960036 q^{8}+\cdots
\end{gathered}
$$

The equality of the Yukawa coupling with the "adjoint" Yukawa coupling, $K(q)=$ $K^{\star}(q)$, is a straight consequence of relation $B_{2} \cdot x=x \cdot \operatorname{adjoint}\left(B_{2}\right)$.

Recalling Batyrev and van Straten [77], and following Morrison [76], do note that one can also write the Yukawa coupling as:

$$
K(q)=\frac{x(q)^{3} \cdot W_{4}^{1 / 2}}{y_{0}^{2}} \cdot\left(\frac{q}{x(q)} \cdot \frac{d x(q)}{d q}\right)^{3}=\frac{W_{4}^{1 / 2}}{y_{0}^{2}} \cdot\left(q \cdot \frac{d x(q)}{d q}\right)^{3},
$$

where $W_{4}$ is the Wronskian (82). From this alternative expression for the Yukawa coupling, valid when the operator is conjugated to its adjoint (see (E.7)), it is obvious that if the analytic series $y_{0}(x)$, as well as the nome (84) are series with integer coefficients, then, the mirror map (85) is also a series with integer coefficients, and, therefore, $y_{0}$ seen as a function of the nome $q$, as well as $x^{3} W_{4}^{1 / 2}$ (since it is a rational function) are also series with integer coefficients. Consequently, the Yukawa coupling is a series with integer coefficients (as a series in $q$ or in $x$ ).

More generally, if one assumes that a linear differential operator has a globally bounded solution-series, one knows that this operator is a $G$-operator, necessarily globally nilpotent, and, consequently, its Wronskian, or the square root of the Wronskian (see $W_{4}^{1 / 2}$ in (87)), will be a $N$-th root of a rational function, and, thus, will correspond to a globally bounded series. Thus, the globally bounded character of the analytic series $y_{0}(x)$ together with the nome, yields the globally bounded character of the mirror map, Yukawa coupling, that we associate with the modularity $†$. In contrast the globally bounded character of the analytic series $y_{0}(x)$, together with the globally bounded character of the Yukawa coupling (seen for instance as a series in $x$ ) does not imply that the nome, or the mirror map, are globally bounded as can be seen on example (59) (see (60) and (61)).

\subsection{An operator non trivially homomorphic to $B_{2}$}

Let us, now, consider the order-four operator

$$
\begin{aligned}
\mathcal{B}_{2}=256 x^{2} \cdot \theta^{2} & (2 \theta+3)(2 \theta+1) \\
& -4 x \cdot(2 \theta+1)(2 \theta-1)\left(5 \theta^{2}-5 \theta+2\right)+(\theta-1)^{4} .
\end{aligned}
$$

This operator is non-trivially $\ddagger$ homomorphic to the Calabi-Yau operator (80):

$$
\mathcal{B}_{2} \cdot x \cdot(2 \theta+1)=x \cdot(2 \theta+1) \cdot B_{2} .
$$

As a consequence of the previous intertwining relation, one immediately finds that the series-solution analytic at $x=0$ of this new MUM operator (88) is nothing but the action of the order-one operator $x \cdot(2 \theta+1)$ on the series (83), and reads:

$$
\begin{aligned}
x \cdot(2 \theta+1)[\mathcal{S}] & =x+24 x^{2}+840 x^{3}+35840 x^{4}+1711080 x^{5}+87329088 x^{6} \\
+ & 4650277632 x^{7}+254905896960 x^{8}+\cdots
\end{aligned}
$$

$\dagger$ Similar results can be found in Delaygue's thesis [98], in a framework where the coefficients of hypergeometric series are ratio of factorials (see Appendix C).

$\ddagger$ The intertwiners between $\mathcal{B}_{2}$ and $B_{2}$ are operators not simple functions. 
It is obviously also a series with integer coefficients (the action of $x \cdot(2 \theta+1)$ on the series with integer coefficients is straightforwardly a series with integer coefficients). More generally, the globally bounded series remain globally bounded series by non trivial operator equivalence, namely homomorphisms between operators (generically the intertwiner operators are not simple functions).

The exterior square of the order-four operator (88) is an order-six operator which is, in fact, the direct sum of an order-five operator $\mathcal{E}_{5}$ and an order-one operator.

Operator $\mathcal{B}_{2}$ is non-trivially homomorphic to its adjoint:

$$
\mathcal{B}_{2} \cdot x^{3} \cdot(2 \theta+3) \cdot(2 \theta+5)=x^{3} \cdot(2 \theta+3) \cdot(2 \theta+5) \cdot \operatorname{adjoint}\left(\mathcal{B}_{2}\right) .
$$

The Yukawa coupling of this order-four operator (88), non-trivially homomorphic to (80), reads:

$$
\begin{aligned}
K(q)= & 1-4 q-140 q^{2}-4040 q^{3}-64436 \frac{q^{4}}{3}+1889332 \frac{q^{5}}{3} \\
& +88331368 \frac{q^{6}}{5}+1652707624 \frac{q^{7}}{9}-69295027684 \frac{q^{8}}{63}+\cdots
\end{aligned}
$$

The Yukawa coupling series (92) is not globally bounded.

The "adjoint Yukawa coupling" of this order-four operator (88) reads:

$$
\begin{aligned}
& K^{\star}(q)=1+12 q+564 q^{2}+20440 q^{3}+865732 q^{4}+37162444 q^{5} \\
& +8255346664 \frac{q^{6}}{5}+1121762648248 \frac{q^{7}}{15}+72336859374772 \frac{q^{8}}{21}+\cdots
\end{aligned}
$$

Again, the adjoint Yukawa coupling series (93) is not globally bounded.

On this example one sees that the Yukawa coupling of two non-trivially homomorphic operators are not necessarily equal. The Yukawa couplings of two homomorphic operators are equal when the two operators are conjugated by a function (trivial homomorphism). The modularity property is not preserved by (non-trivial) operator equivalence: it may depend on a condition that the exterior square of the order-four operators is of order five. The Calabi-Yau property is not preserved by operator equivalence.

To sum-up: All these examples show that the integrality (globally bounded series) is far from identifying with modularity.

\section{Conclusion}

Seeking for the linear differential operators for the $\chi^{(n)}$ 's, we discovered, some years ago, that they were Fuchsian operators [11, 13], and, in fact, "special" Fuchsian operators, namely Fuchsian operators with rational exponents for all their singularities, and with Wronskians that are $N$-th roots of rational functions. Then we discovered that they were $G$-operators (or equivalently globally nilpotent [27]), and more recently, we accumulated results [31] indicating that they are "special" $G$ operators. There are, in fact, two quite different kinds of "special features" of these $G$-operators. On one side, we have the fact that one of their solutions is not only $G$-series, but is a globally bounded series. This special character has been addressed in this very paper, and we have seen that, in fact, this "integrality property [99]" is a consequence of quite general mathematical assumptions often satisfied in physics (the integrand is not only algebraic but has an expansion at the origin of the form $\dagger$ (35).

$\dagger$ Puiseux series are excluded. 
However, we have also seen another special property of these $G$-operators, namely the fact that they seem to be quite systematically homomorphic to their adjoints [31]. We will show, in a forthcoming publication, that this last property amounts, on the associated linear differential systems, to having special differential Galois groups, and that their exterior or symmetric squares, have rational solutions. This last property is a property of a more "physical" nature than the previous one, related to an underlying Hamiltonian structure [100], or as this is the case, for instance in the Ising model, related to the underlying isomonodromic structure in the problem, which yields the occurrence of some underlying Hamiltonian structure [100]. In general the integrality of $G$-operators does not imply the operator to be homomorphic to its adjoint, and conversely being homomorphic to its adjoint does not implył integrality (and even does not imply $\dagger$ the operator to be Fuchsian). Interestingly, the $\chi^{(n)}$ 's, as well as many important problems of theoretical physics, correspond to $G$-operators that present these two complementary "special characters" (integrality and, up to homomorphisms, self-adjointness), and, quite often, this is seen in the framework of the emergence of "modularity".

Nomes, mirror maps, and Yukawa couplings are not D-finite functions: they are solutions of quite involved non-linear (higher order Schwarzian) ODEs (see for instance Appendix D in [27]). Therefore, the question of the series integrality of the nomes, mirror maps, Yukawa couplings, and other pullback-invariants (see Appendix E) requires to address the very difficult question of series-integrality for (involved) nonlinear ODEs. Note, however, as seen in Section 8.1, in particular in (87), that the integrality of the series $y_{0}(x)$ and of the nome $q(x)$ are sufficient to ensure, provided the operator is conjugated to its adjoint (see (E.7)), the integrality of the other quantities such as the Yukawa coupling, mirror maps. However the integrality of the nome remains an involved problem. These questions will certainly remain open for some time.

In contrast, and more modestly, we have shown that a very large sets of problems in mathematical physics (see sections (3.4), (4) and (5.1)) actually corresponds to diagonals of rational functions. In particular, we have been able to show that the $\chi^{(n)}$ 's $n$-fold integrals of the susceptibility of the two-dimensional Ising model are actually diagonals of rational functions for any value of the integer $n$, thus proving that the $\chi^{(n)}$ 's are globally bounded for any value of the integer $n$. As can be seen in the "ingredients" of our simple demonstration (see (3.4)), no elliptic curves, and their modular forms [102], no Calabi-Yau [103], or Frobenius manifolds [100], or Shimura curves, or arithmetic lattice assumption $[104,105]$ is required to prove the result. We just need to have an $n$-fold integral such that its integrand is not only algebraic, but has an expansion at the origin of the form (35).

The integrality of all the $\chi^{(n)}$ 's, consequence of the remarkable result that the all $\chi^{(n)}$ 's are diagonal of rational functions, raises the question of the modularity of the $\chi^{(n)}$ 's. Now, the full susceptibility can, formally, be seen as the diagonal of an infinite sum of rational functions. This also raises the question of defining, and addressing, modularity for non-holonomic functions§ like the full susceptibility.

$\ddagger$ See Appendix M and Appendix O in [19] which give an example of a (hypergeometric) family of order-four operators satisfying the Calabi-Yau condition that their exterior square is of order five, and, even, a family of self-adjoint order-four operators, the corresponding hypergeometric solution-series being not globally bounded.

$\dagger$ For instance the operator $D_{x}^{n}-x D_{x}-1 / 2$ (see page 74 of [101]) with an irregular singularity is self-adjoint.

$\S$ Along this line, recall Chazy's equations [106] and their (circle) natural boundaries, and, especially, 
Acknowledgments We would like to thank A. Enge and F. Morain for interesting and detailed discussions on Fricke and Atkin-Lehner involutions. S. B. would like to thank the LPTMC and the CNRS for kind support. A. B. was supported in part by the Microsoft Research-Inria Joint Centre. As far as physicists authors are concerned, this work has been performed without any support of the ANR, the ERC or the MAE.

\section{Appendix A. Modular forms and series integrality}

First example: The generating function of the integers

$$
\begin{aligned}
\sum_{k=0}^{n}\left(\begin{array}{l}
n \\
k
\end{array}\right)^{2} & \cdot\left(\begin{array}{c}
2 k \\
k
\end{array}\right) \cdot\left(\begin{array}{c}
2 n-2 k \\
n-k
\end{array}\right) \\
& =\left(\begin{array}{c}
2 n \\
n
\end{array}\right) \cdot{ }_{2} F_{1}\left(\left[\frac{1}{2},-n,-n,-n\right],\left[1,1,-\frac{2 n-1}{2}\right] ; 1\right),
\end{aligned}
$$

is nothing else but the expansion of the square of a HeunG function

$$
\begin{aligned}
\operatorname{Heun} G(4, & \left.\frac{1}{2}, \frac{1}{2}, \frac{1}{2}, 1, \frac{1}{2} ; 16 \cdot x\right)=1+2 x+12 x^{2}+104 x^{3} \\
& +1078 x^{4}+12348 x^{5}+150528 x^{6}+1914432 x^{7}+\cdots
\end{aligned}
$$

solution of the order-two operator

$$
H_{\text {diam }}=\theta^{2}-2 \cdot x \cdot\left(10 \theta^{2}+5 \theta+1\right)+16 x^{2} \cdot(2 \theta+1)^{2} .
$$

which corresponds to the diamond lattice [43]. This HeunG function (A.2) is actually a modular form which can be written in two different ways:

$$
\begin{aligned}
\text { HeunG } & \left(4, \frac{1}{2}, \frac{1}{2}, \frac{1}{2}, 1, \frac{1}{2} ; 16 x\right) \\
& =(1-4 x)^{-1 / 2} \cdot{ }_{2} F_{1}\left(\left[\frac{1}{6}, \frac{1}{3}\right],[1] ; \frac{108 x^{2}}{(1-4 x)^{3}}\right) \\
& =(1-16 x)^{-1 / 2} \cdot{ }_{2} F_{1}\left(\left[\frac{1}{6}, \frac{1}{3}\right],[1] ;-\frac{108 x}{(1-16 x)^{3}}\right) .
\end{aligned}
$$

These two pullbacks are related by an "Atkin" involution $x \leftrightarrow 1 / 64 / x$. The associated modular curve, relating these two pullbacks (A.4) yielding the modular curve:

$$
\begin{aligned}
4 \cdot y^{3} z^{3} & -12 y^{2} z^{2} \cdot(y+z)+3 y z \cdot\left(4 y^{2}+4 z^{2}-127 y z\right) \\
& -4 \cdot(y+z) \cdot\left(y^{2}+z^{2}+83 y z\right)+432 y z=0,
\end{aligned}
$$

which is $(y, z)$-symmetric and is exactly the rational modular curve in eq. (27) already found for the order-three operator $F_{3}$ in [31] for the five-particle contribution $\tilde{\chi}^{(5)}$ of the magnetic susceptibility of the Ising model.

This result in $[41,43]$ can be rephrased as follows. One introduces the order-three operator which has the following ${ }_{3} F_{2}$ solution

$$
\frac{1}{\left(4-x^{2}\right)^{3}} \cdot{ }_{3} F_{2}\left(\left[\frac{1}{3}, \frac{1}{2}, \frac{2}{3}\right],[1,1], \frac{27 x^{4}}{\left(4-x^{2}\right)^{3}}\right)
$$

Harnad and McKay paper [107] on modular solutions to equations of generalized Halphen type. 
associated with the Green function of the diamond lattice. Along a modular form line lets us note that this hypergeometric function actually has two pullbacks:

$$
\begin{aligned}
{ }_{3} F_{2}\left(\left[\frac{1}{3},\right.\right. & \left.\left.\frac{1}{2}, \frac{2}{3}\right],[1,1], \frac{27 x^{4}}{\left(4-x^{2}\right)^{3}}\right) \\
& =\frac{x^{2}-4}{4 \cdot\left(x^{2}-1\right)} \cdot{ }_{3} F_{2}\left(\left[\frac{1}{3}, \frac{1}{2}, \frac{2}{3}\right],[1,1], \frac{27 x^{2}}{4 \cdot\left(x^{2}-1\right)^{3}}\right) .
\end{aligned}
$$

These two pullbacks related by the "Atkin" involution $x \rightarrow 2 / x$ :

$$
u(x)=\frac{27 x^{4}}{\left(4-x^{2}\right)^{3}}, \quad v(x)=u\left(\frac{2}{x}\right)=\frac{27 x^{2}}{4 \cdot\left(x^{2}-1\right)^{3}},
$$

corresponding, again, to the modular curve (A.5).

Second example. The HeunG function

$$
\begin{aligned}
& \text { Heun } G(-3,0,1 / 2,1,1,1 / 2 ; 12 \cdot x) \\
& =(1+4 x)^{-1 / 4} \cdot \operatorname{Heun} G\left(4, \frac{1}{2}, \frac{1}{2}, \frac{1}{2}, 1, \frac{1}{2}, \frac{16 x}{1+4 x}\right) \\
& =1+6 x^{2}+24 x^{3}+252 x^{4}+2016 x^{5}+19320 x^{6}+183456 x^{7} \\
& +1823094 x^{8}+18406752 x^{9}+189532980 x^{10}+\cdots
\end{aligned}
$$

is solution of the order-two operator

$$
H_{f c c}=\theta^{2}-2 x \cdot \theta \cdot(4 \theta+1)-24 \cdot x^{2} \cdot(2 \theta+1) \cdot(\theta+1),
$$

The square of (A.9) is actually the solution of an order-three operator (see equation (19) in [43]) emerging for lattice Green functions of the face-centred cubic (fcc) lattice which is thus the symmetric square of (A.10). This hypergeometric function with a polynomial pull-back can also be written:

$$
\begin{aligned}
H e u n G & (-3,0,1 / 2,1,1,1 / 2 ; 12 \cdot x) \\
& ={ }_{2} F_{1}\left(\left[\frac{1}{6}, \frac{1}{3}\right],[1] ; 108 \cdot x^{2} \cdot(1+4 x)\right) \\
& =(1-12 x)^{-1 / 2} \cdot{ }_{2} F_{1}\left(\left[\frac{1}{6}, \frac{1}{3}\right],[1] ;-\frac{108 \cdot x \cdot(1+4 x)^{2}}{(1-12 x)^{3}}\right),
\end{aligned}
$$

where the involution $x \leftrightarrow-1 / 4 \cdot(1+4 x) /(1-12 x)$ takes place. The modular curve relating these two pullbacks reads exactly the rational curve (A.5) already obtained in [31].

Third example. The HeunG function HeunG $(1 / 9,1 / 12,1 / 4,3 / 4,1,1 / 2 ; 4 x)$ is solution of the order-two operator corresponding to the simple cubic lattice Green function

$$
H_{s c}=\theta^{2}-x \cdot\left(40 \theta^{2}+20 \theta+3\right)+9 \cdot x^{2} \cdot(4 \theta+3) \cdot(4 \theta+1) .
$$

The square of this HeunG function is a series with integer coefficients which identifies with the Hadamard product of $(1-4 x)^{-1 / 2}$ with a modular form :

$$
\begin{aligned}
\text { HeunG }(1 / 9,1 / 12,1 / 4,3 / 4,1,1 / 2 ; 4 x)^{2} & \text { (A.12) } \\
= & (1-4 x)^{-1 / 2} \star \operatorname{HeunG}(1 / 9,1 / 3,1,1,1,1 ; x) \\
= & \begin{aligned}
1 \quad & +6 x+90 x^{2}+1860 x^{3}+44730 x^{4}+1172556 x^{5}+32496156 x^{6} \\
& +936369720 x^{7}+27770358330 x^{8}+842090474940 x^{9}+\cdots
\end{aligned}
\end{aligned}
$$

The HeunG function $\operatorname{Heun} G(1 / 9,1 / 12,1 / 4,3 / 4,1,1 / 2 ; 4 x)$ is globally bounded: the series of HeunG $(1 / 9,1 / 12,1 / 4,3 / 4,1,1 / 2 ; 8 x)$ is a series with integer coefficients. 
One can also write this HeunG function in terms of a ${ }_{2} F_{1}([1 / 6,1 / 3],[1], x)$ hypergeometric function up to a simple algebraic pullback (with a square root), or in terms of a ${ }_{2} F_{1}([1 / 8,3 / 8],[1], x)$ hypergeometric function:

$$
\begin{aligned}
& \operatorname{Heun} G(1 / 9,1 / 12,1 / 4,3 / 4,1,1 / 2 ; 4 x)=C_{2}^{1 / 4} \cdot{ }_{2} F_{1}\left([1 / 8,3 / 8],[1] ; P_{2}\right), \quad \text { with: } \\
& C_{2}=\frac{1}{9 \cdot(1+12 x)^{2}} \cdot\left(5-36 x+4 \cdot(1-36 x)^{1 / 2}\right), \quad P_{2}=\frac{128 \cdot x}{(1+12 x)^{4}} \cdot p_{2}, \\
& p_{2}=\left(1-42 x+352 x^{2}-288 x^{3}\right)+(1-4 x) \cdot(1-20 x) \cdot(1-36 x)^{1 / 2} .
\end{aligned}
$$

Do note that taking the Galois conjugate (changing $(1-36 x)^{1 / 2}$ into $\left.-(1-36 x)^{1 / 2}\right)$ gives the series expansion of $3^{-1 / 2} \cdot \operatorname{Heun} G(1 / 9,1 / 12,1 / 4,3 / 4,1,1 / 2 ; 4 x)$. This shows that there exists an identity for ${ }_{2} F_{1}([1 / 8,3 / 8],[1], x)$ with two different pullbacks, namely the previous $P_{2}$ and its Galois conjugate, these two pullbacks being related by a (symmetric genus zero) modular curve:

$$
\begin{aligned}
& 5308416 \cdot y^{4} z^{4}+442368 \cdot y^{3} z^{3} \cdot(y+z)+512 y^{2} z^{2} \cdot\left(27 y^{2}+27 z^{2}-27374 x y\right) \\
& \quad+192 y z \cdot(y+z) \cdot\left(y^{2}+z^{2}+10718 y z\right)+y^{4}+z^{4}+3622662 y^{2} z^{2} \\
& \left.\quad-19332 \cdot y z \cdot\left(y^{2}+z^{2}\right)+79872 \cdot y z \cdot(y+z)-65536 \cdot y z=0 . \quad \text { (A.1 }\right)
\end{aligned}
$$

Revisiting the examples. In a recent paper [44] corresponding to spanning tree generating functions and Mahler measures, a result from Rogers (equation (36) in [44]) is given where the two following ${ }_{5} F_{4}$ hypergeometric functions take place:

$$
\begin{aligned}
& { }_{5} F_{4}\left(\left[\frac{5}{4}, \frac{3}{2}, \frac{7}{4}, 1,1\right],[2,2,2,2], \frac{256 x^{3}}{9 \cdot(x+3)^{4}}\right), \\
& { }_{5} F_{4}\left(\left[\frac{5}{4}, \frac{3}{2}, \frac{7}{4}, 1,1\right],[2,2,2,2], \frac{256 x}{9 \cdot(1+3 x)^{4}}\right) .
\end{aligned}
$$

The corresponding order-five linear differential operators (annihilating these two ${ }_{5} F_{4}$ hypergeometric functions) are actually homomorphic (the intertwiners being orderfour operators). The relation between these two pullbacks $y=256 x^{3} / 9 /(x+3)^{4}$ and $z=256 x / 9 /(1+3 x)^{4}$, remarkably gives, again, the previous $(y, z)$-symmetric modular curve (A.13).

The order-five linear differential operator, corresponding to the first ${ }_{5} F_{4}$ hypergeometric function, factorizes in an order-one operator, an order-three operator and an order-one operator, the order-three operator being, in fact, exactly the symmetric square of an order-two operator:

$$
L_{1} \cdot \operatorname{Sym}^{2}\left(W_{2}\right) \cdot \frac{x^{4}}{(x-9)(x+3)^{4}} \cdot R_{1},
$$

where the order-one operators read respectively

$$
L_{1}=D_{x}-\frac{d}{d x} \ln \left(\frac{x-9}{\left(9 x^{2}+14 x+9\right) \cdot(x+3)^{4}}\right), \quad R_{1}=D_{x}-\frac{d}{d x} \ln \left(\frac{(x+3)^{4}}{x^{3}}\right),
$$

and where the order-two operator $W_{2}$ reads:

$$
W_{2}=D_{x}^{2}+3 \frac{\left(6 \cdot x^{2}+7 x+3\right)}{\left(9 x^{2}+14 x+9\right) \cdot x} \cdot D_{x}+\frac{3}{4} \cdot \frac{3 x+2}{\left(9 x^{2}+14 x+9\right) \cdot x} .
$$

We have a similar result for the order-five linear differential operator corresponding to the second ${ }_{5} F_{4}$ hypergeometric function. 
Another solution of this order-five linear differential operator reads:

$$
\frac{(x+3)^{4}}{x^{3}} \cdot \int \frac{x-9}{(x+3) \cdot x} \cdot{ }_{3} F_{2}\left(\left[\frac{1}{4}, \frac{1}{2}, \frac{3}{4}\right],[1,1], \frac{256 x^{3}}{9 \cdot(x+3)^{4}}\right) \cdot d x .
$$

The expansion of the ${ }_{3} F_{2}$ hypergeometric function in (A.16) is globally bounded (change $x \rightarrow 9 x$ to get a series with integer coefficients).

Recalling the two previous pullbacks we have, in fact, the following identity:

$$
\begin{aligned}
3 \cdot(1+3 x) \cdot{ }_{3} F_{2}\left(\left[\frac{1}{4}, \frac{1}{2}, \frac{3}{4}\right],[1,1], \frac{256 x^{3}}{9 \cdot(x+3)^{4}}\right) \\
=(x+3) \cdot{ }_{3} F_{2}\left(\left[\frac{1}{4}, \frac{1}{2}, \frac{3}{4}\right],[1,1], \frac{256 x}{9 \cdot(1+3 x)^{4}}\right) .
\end{aligned}
$$

However this ${ }_{3} F_{2}$ hypergeometric function is nothing but the square of a ${ }_{2} F_{1}$ hypergeometric function

$$
{ }_{3} F_{2}\left(\left[\frac{1}{4}, \frac{1}{2}, \frac{3}{4}\right],[1,1], x\right)={ }_{2} F_{1}\left(\left[\frac{1}{8}, \frac{3}{8}\right],[1], x\right)^{2} .
$$

Thus, the previous identity (A.17) is nothing but the identity on a ${ }_{2} F_{1}$ hypergeometric function with two different pullbacks:

$$
\begin{aligned}
(1+3 x)^{1 / 2} & \cdot{ }_{2} F_{1}\left(\left[\frac{1}{8}, \frac{3}{8}\right],[1], \frac{256 x^{3}}{9 \cdot(x+3)^{4}}\right) \\
& =\left(1+\frac{x}{3}\right)^{1 / 2} \cdot{ }_{2} F_{1}\left(\left[\frac{1}{8}, \frac{3}{8}\right],[1], \frac{256 x}{9 \cdot(1+3 x)^{4}}\right) .
\end{aligned}
$$

The expansion of (A.19) is globally bounded. One gets a series with positive integer coefficients using the simple rescaling $x \rightarrow 36 \cdot x$. Note that the two pullbacks can be exchanged by the simple "Atkin" involution $x \leftrightarrow 1 / x$, being related by the modular curve occurring for the simple cubic lattice, namely (A.13). in [44].

We have a similar result for the other ${ }_{5} F_{4}$ hypergeometric functions popping out

For instance, for the diamond lattice one gets an expression (see eq. (50) in [44]) where the two following ${ }_{5} F_{4}$ hypergeometric functions take placeł:

$$
\begin{aligned}
& { }_{5} F_{4}\left(\left[\frac{5}{3}, \frac{3}{2}, \frac{4}{3}, 1,1\right],[2,2,2,2], \frac{-27 x^{2}}{4 \cdot\left(1-x^{2}\right)^{3}}\right), \\
& { }_{5} F_{4}\left(\left[\frac{5}{3}, \frac{3}{2}, \frac{4}{3}, 1,1\right],[2,2,2,2], \frac{27 x^{4}}{\left(4-x^{2}\right)^{3}}\right) .
\end{aligned}
$$

These two pullbacks can be exchanged by the simple "Atkin" involution $x \leftrightarrow 2 / x$. These two pullbacks have been seen to be related by the (genus-zero) $(y, z)$-symmetric modular curve (A.5):

$$
\begin{array}{r}
4 y^{3} z^{3}-12 y^{2} z^{2} \cdot(y+z)+3 y z\left(4 y^{2}+4 z^{2}-127 y z\right) \\
-4 \cdot(y+z) \cdot\left(y^{2}+z^{2}+83 y z\right)+432 y z=0 .
\end{array}
$$

Similarly to (A.17) we have an identity between two ${ }_{3} F_{2}$ hypergeometric functions (namely ${ }_{3} F_{2}([2 / 3,1 / 2,1 / 3],[1,1], z)$ ) with the two pullbacks (A.20), and these ${ }_{3} F_{2}$

$\ddagger$ Note a small misprint in eq. (50) of [44]: one should read $-27 z^{2} / 4 /\left(1-z^{2}\right)^{3}$ instead of $-27 z^{4} / 4 /\left(1-z^{2}\right)^{3}$. 
hypergeometric functions being the square of ${ }_{2} F_{1}$ hypergeometric functions, one finds that the "deus ex machina" is the identity similar to (A.19):

$$
\begin{aligned}
\left(1-x^{2}\right)^{1 / 2} & \cdot{ }_{2} F_{1}\left(\left[\frac{1}{3}, \frac{1}{6}\right],[1], \frac{27 x^{4}}{\left(4-x^{2}\right)^{3}}\right) \\
& =\left(1-\frac{x^{2}}{4}\right)^{1 / 2} \cdot{ }_{2} F_{1}\left(\left[\frac{1}{3}, \frac{1}{6}\right],[1], \frac{-27 x^{2}}{4 \cdot\left(1-x^{2}\right)^{3}}\right) .
\end{aligned}
$$

The series expansion of (A.22) is globally bounded. Rescaling the $x$ variable as $x \rightarrow 4 x$, the series expansion becomes a series with positive integer coefficients (up to the first constant term).

For the face-centred cubic lattice one gets an expression (see eq. (52) in [44]) where the two following ${ }_{5} F_{4}$ hypergeometric functions take placet:

$$
\begin{aligned}
& { }_{5} F_{4}\left(\left[\frac{5}{3}, \frac{3}{2}, \frac{4}{3}, 1,1\right],[2,2,2,2], \frac{x \cdot(x+3)^{2}}{(x-1)^{3}}\right), \\
& { }_{5} F_{4}\left(\left[\frac{5}{3}, \frac{3}{2}, \frac{4}{3}, 1,1\right],[2,2,2,2], \frac{x^{2} \cdot(x+3)}{4}\right) .
\end{aligned}
$$

This example is nothing but the previous diamond lattice example (A.20) with the change of variable $x \rightarrow-3 x^{2} /\left(x^{2}-4\right)$ in (A.23). Therefore, the two pullbacks in (A.23) are, again, related by the modular curve (A.5). The two pullbacks in (A.23) can actually be seen directly in the following identity (equivalent to (A.22)):

$$
{ }_{2} F_{1}\left(\left[\frac{1}{3}, \frac{1}{6}\right],[1], \frac{x \cdot(x+3)^{2}}{(x-1)^{3}}\right)=\left(1-x^{2}\right)^{1 / 2} \cdot{ }_{2} F_{1}\left(\left[\frac{1}{3}, \frac{1}{6}\right],[1], \frac{x^{2} \cdot(x+3)}{4}\right) \text {. }
$$

Finally, the equation (17) of [44] on Mahler measures, the two following ${ }_{4} F_{3}$ hypergeometric functions take place:

$$
\begin{aligned}
& { }_{4} F_{3}\left(\left[\frac{5}{3}, \frac{4}{3}, 1,1\right],[2,2,2], \frac{27 x}{(x-2)^{3}}\right), \\
& { }_{4} F_{3}\left(\left[\frac{5}{3}, \frac{4}{3}, 1,1\right],[2,2,2], \frac{27 x^{2}}{(x+4)^{3}}\right) .
\end{aligned}
$$

These two previous pullbacks can be exchanged by an "Atkin" involution $x \leftrightarrow-8 / x$ and are related by the (genus-zero) $(y, z)$-symmetric modular curve:

$$
\begin{aligned}
8 y^{3} z^{3}-12 y^{2} z^{2} \cdot(y+z)+3 y z \cdot\left(2 y^{2}+2 z^{2}+13 y z\right) & \\
- & (y+z) \cdot\left(y^{2}+z^{2}+29 y z\right)+27 y z=0 .
\end{aligned}
$$

The underlying identity on ${ }_{2} F_{1}$ hypergeometric functions with the two pullbacks (A.24) read:

$$
\begin{aligned}
-2 \cdot(x-2) \cdot{ }_{2} F_{1}\left(\left[\frac{1}{3}, \frac{2}{3}\right],[1], \frac{27 x^{2}}{(x+4)^{3}}\right) \\
=(x+4) \cdot{ }_{2} F_{1}\left(\left[\frac{1}{3}, \frac{2}{3}\right],[1], \frac{27 x}{(x-2)^{3}}\right) .
\end{aligned}
$$

The series expansion of (A.26) is globally bounded. Rescaling the $x$ variable as $x \rightarrow-8 x$, the series expansion becomes a series with positive integer coefficients.

$\dagger$ There is one more misprint in [44]: the pullback $-x(x+3) /(x-1)^{3}$ must be changed into $x(x+3) /(x-1)^{3}$. 


\section{Appendix B. Seeking for the "minimal" rational function}

The effective calculations of section (4.2) guarantee to obtain an explicit expression for the rational function associated with (40), however the rational function is far from being unique. Recalling the well-known Apéry series $\mathcal{A}(x)$, and its rewriting due to Strehl and Schmidt [85, 86, 87],

$$
\begin{gathered}
\mathcal{A}(x)=\sum_{n=0}^{\infty} \sum_{k=0}^{n}\left(\begin{array}{l}
n \\
k
\end{array}\right)^{2}\left(\begin{array}{c}
n+k \\
k
\end{array}\right)^{2} \cdot x^{n}=\sum_{n=0}^{\infty} \sum_{k=0}^{n} \sum_{j=0}^{k}\left(\begin{array}{l}
n \\
k
\end{array}\right)\left(\begin{array}{c}
n+k \\
k
\end{array}\right)\left(\begin{array}{l}
k \\
j
\end{array}\right)^{3} \cdot x^{n} \\
=\quad 1+5 x+73 x^{2}+1445 x^{3}+33001 x^{4}+\cdots,
\end{gathered}
$$

$\mathcal{A}(x)$ is known to be the diagonal of the rational function in five variables $1 / R_{1} / R_{2}$ where $R_{1}, R_{2}$ read [60]:

$$
R_{1}=1-z_{0}, \quad R_{2}=\left(1-z_{1}\right)\left(1-z_{2}\right)\left(1-z_{3}\right)\left(1-z_{4}\right)-z_{0} z_{1} z_{2},
$$

as well as the diagonal of the rational function in five variables $1 / Q_{1} / Q_{2}$ where $Q_{1}, Q_{2}$ read $[61,45]$ :

$$
Q_{1}=1-z_{1} z_{2} z_{3} z_{4}, \quad Q_{2}=\left(1-z_{3}\right)\left(1-z_{4}\right)-z_{0} \cdot\left(1+z_{1}\right)\left(1+z_{2}\right),
$$

and also the diagonal of the rational function in six variables $1 / P_{1} / P_{2} / P_{3}$ where $P_{1}, P_{2}, P_{3}$ read [60]:

$$
P_{1}=1-z_{0} z_{1}, \quad P_{2}=1-z_{2}-z_{3}-z_{0} z_{2} z_{3}, \quad P_{3}=1-z_{4}-z_{5}-z_{1} z_{4} z_{5} .
$$

A yet different diagonal representation for the Apéry series, due to Delayguet, is provided by the diagonal of the rational function in eight variables:

$\frac{1}{\left(1-z_{4} z_{5} z_{6} z_{7}\right) \cdot\left(1-z_{0} \cdot\left(1+z_{4}\right)\right) \cdot\left(1-z_{1} \cdot\left(1+z_{5}\right)\right) \cdot\left(1-z_{2}-z_{6}\right) \cdot\left(1-z_{3}-z_{7}\right)}$.

Calculations similar to (39) on these new binomial expressions provide two new rational functions such that (B.1) can be written as the diagonal of one of these two rational functions. One is a rational function of five variables, of the form $1 / Q_{1}^{(5)} / Q_{2}^{(5)}$

$$
\begin{aligned}
& Q_{1}^{(5)}=1-z_{0} z_{1} z_{2} z_{3} z_{4} \cdot\left(1+z_{1}\right)\left(1+z_{2}\right)\left(1+z_{3}\right)\left(1+z_{4}\right), \\
& Q_{2}^{(5)}=1-z_{0} \cdot\left(1+z_{1}\right)\left(1+z_{2}\right)\left(1+z_{3}\right)^{2}\left(1+z_{4}\right)^{2},
\end{aligned}
$$

and the other one, is a rational function of six variables, of the form $1 / Q_{1}^{(6)} / Q_{2}^{(6)} / Q_{3}^{(6)}$

$$
\begin{aligned}
& Q_{1}^{(6)}=1-z_{0} z_{3} z_{4} z_{5} \cdot\left(1+z_{1}\right)\left(1+z_{2}\right)^{2}\left(1+z_{3}\right)\left(1+z_{4}\right)\left(1+z_{5}\right), \\
& Q_{2}^{(6)}=1-z_{0} z_{1} z_{2} z_{3} z_{4} z_{5} \cdot\left(1+z_{1}\right)\left(1+z_{2}\right), \\
& Q_{3}^{(6)}=1-z_{0} \cdot\left(1+z_{1}\right)\left(1+z_{2}\right)^{2}\left(1+z_{3}\right)\left(1+z_{4}\right)\left(1+z_{5}\right) .
\end{aligned}
$$

\section{Appendix C. Hypergeometric series with coefficients ratio of factorials}

As a consequence of the classification by Beukers and Heckman [91] of all algebraic ${ }_{n} F_{n-1}$ 's, the ${ }_{8} F_{7}$ hypergeometric series

$$
{ }_{8} F_{7}\left(\left[\frac{1}{30}, \frac{7}{30}, \frac{11}{30}, \frac{13}{30}, \frac{17}{30}, \frac{19}{30}, \frac{23}{30}, \frac{29}{30}\right],\left[\frac{1}{5}, \frac{2}{5}, \frac{3}{5}, \frac{4}{5}, \frac{1}{2}, \frac{2}{3}, \frac{1}{3}\right], 2^{14} 3^{9} 5^{5} x\right),
$$

$\dagger$ Personal communication. 
has integer coefficients, and is an algebraic function. The Galois group belonging to this function is the Weyl group $W\left(E_{8}\right)$ which has 696729600 elements [108]. It is an algebraic series of degree 483840 . More precisely, it was noticed by RodriguezVillegas [109] that the previous power series reads:

$$
\sum_{n=0}^{\infty} \frac{(30 n) ! n !}{(15 n) !(10 n) !(6 n) !} \cdot x^{n}
$$

which is closely related to the series introduced by Chebyshev in his work [110] on the distribution of prime numbers to establish the estimate [19] on the prime counting function $\pi(x)$.

Considering hypergeometric series such that their coefficients are ratio of factorials, reference [109] gives the conditions of these factorials for the hypergeometric series to be algebraic (all the coefficients are thus integers). A simple example is, for instance the algebraic function:

$$
{ }_{3} F_{2}\left(\left[\frac{1}{4}, \frac{1}{2}, \frac{3}{4}\right],\left[\frac{1}{3}, \frac{2}{3}\right] ; \frac{2^{8}}{3^{3}} \cdot x\right)=\sum_{n=0}^{\infty}\left(\begin{array}{c}
4 n \\
n
\end{array}\right) \cdot x^{n} .
$$

Along this line it is worth recalling Delaygue's Thesis [98] (see also Bober [111]) which gives some results\# for series expansions $\Phi$ such that their coefficients are ratio of factorials, namely ${ }_{2} F_{1}([1 / 3,2 / 3],[1] ; 27 x)$, and ${ }_{4} F_{3}([1 / 2,1 / 2,1 / 2,1 / 2],[1,1,1] ; 256 x)$, giving respectively the series

$$
\begin{gathered}
\sum_{n=0}^{\infty} \frac{(3 n) !}{(n !)^{3}} \cdot x^{n}, \quad \sum_{n=0}^{\infty} \frac{((2 n) !)^{4}}{(n !)^{8}} \cdot x^{n}, \quad \text { and: } \\
{ }_{4} F_{3}\left(\left[\frac{1}{2}, \frac{1}{2}, \frac{1}{6}, \frac{5}{6}\right],[1,1,1] ; 2^{8} 3^{3} \cdot x\right)=\sum_{n=0}^{\infty} \frac{(6 n) !(2 n) !}{(3 n) !(n !)^{5}} \cdot x^{n} .
\end{gathered}
$$

These ratio of factorials are all integer numbers.

\section{Appendix D. Proof of integrality of series (43)}

Let us sketch the proof of the integrality of series (43), namely, the integrality of coefficients (45). For each power of the integer number $q=p^{n}$ a term like $4+9 n$ is periodically divisible (period $p$ ) by $q$. In order to have the ratio (46) be an integer, one needs the numerator to be divisible by this factor $q$ before the denominator. The case $p=3$ is an easy one. The other prime $p$ do not divide 9 . One needs to find the first case of divisibility, namely the first integer $n$ such that $4+9 n=k q$ (this corresponds to the smallest $k$ ). If $d q=1, \bmod .9$ then $k=4 d$, mod.9. In other words, the smallest $k$ is the rest of $4 d, \bmod .9$. Consequently, we have replaced the calculations, for every integer $q$, by a finite set of calculations for $d=1,2,4,5,7,8$. Let us use this approach for the ratio (46).

Remark: The terms $n+1$ are always the last to be divisible by $q$. Hence, one can forget its factors. However, one needs as many factors at the numerator than at

$\sharp$ Necessary and sufficient conditions for the integrality of the mirror maps series.

I These series are not algebraic functions. 
the denominator. For the other terms, the following table of the rest of $d \cdot a$ gives the complete proof:

$\begin{array}{lllllll}. & 1 & 2 & 4 & 5 & 7 & 8 \\ 1 & 1 & 2 & 4 & 5 & 7 & 8 \\ 4 & 4 & 8 & 7 & 2 & 1 & 5 \\ 5 & 5 & 1 & 2 & 7 & 8 & 4 \\ 3 & 3 & 6 & 3 & 6 & 3 & 6\end{array}$

One finds out that this is always a factor of the numerator, before the occurrence of a factor at the denominator.

\section{Appendix E. Yukawa couplings}

Appendix E.1. Yukawa couplings as ratio of determinants

Consider an order-four MUM linear differential operator. Let us introduce the determinantal variables $W_{m}=\operatorname{det}\left(M_{m}\right)$ which are the determinantsł of the following $m \times m$ matrices $M_{m}, m=1, \ldots, 4$, with entries expressed in terms of derivatives of the four solutions $y_{0}(x), y_{1}(x), y_{2}(x)$ and $y_{3}(x)$ of the MUM linear differential operator (see Section (4.1) for the definitions). One takes $W_{1}(x)=y_{0}(x)$ and:

$$
\begin{aligned}
& M_{2}=\left[\begin{array}{cc}
y_{0} & y_{1} \\
y_{0}^{\prime} & y_{1}^{\prime}
\end{array}\right], \quad M_{3}=\left[\begin{array}{ccc}
y_{0} & y_{1} & y_{2} \\
y_{0}^{\prime} & y_{1}^{\prime} & y_{2}^{\prime} \\
y_{0}^{\prime \prime} & y_{1}^{\prime \prime} & y_{2}^{\prime \prime}
\end{array}\right], \quad M_{4}=\left[\begin{array}{cccc}
y_{0} & y_{1} & y_{2} & y_{3} \\
y_{0}^{\prime} & y_{1}^{\prime} & y_{2}^{\prime} & y_{3}^{\prime} \\
y_{0}^{\prime \prime} & y_{1}^{\prime \prime} & y_{2}^{\prime \prime} & y_{3}^{\prime \prime} \\
y_{0}^{\prime \prime \prime} & y_{1}^{\prime \prime \prime} & y_{2}^{\prime \prime \prime} & y_{3}^{\prime \prime \prime}
\end{array}\right], \\
& \text { where: } \quad y_{i}^{\prime}=\frac{d}{d x} y_{i}, \quad y_{i}^{\prime \prime}=\frac{d^{2}}{d x^{2}} y_{i}, \quad y_{i}^{\prime \prime \prime}=\frac{d^{3}}{d x^{3}} y_{i} . \quad
\end{aligned}
$$

Since $q$ is equal to $q=\exp \left(y_{1} / y_{0}\right)$, and its derivative verifies

$$
q \cdot \frac{d}{d q}=\frac{W_{1}^{2}}{W_{2}} \cdot \frac{d}{d x}=\frac{y_{0}^{2}}{W_{2}} \cdot \frac{d}{d x}
$$

we have that

$$
\left(q \cdot \frac{d}{d q}\right)^{2}=\frac{y_{0}^{4}}{W_{2}^{2}} \cdot \frac{d^{2}}{d x^{2}}+2 \frac{y_{0}^{3}}{W_{2}^{2}} \frac{d y_{0}}{d x} \cdot \frac{d}{d x}-\frac{y_{0}^{4}}{W_{2}^{3}} \frac{d W_{2}}{d x} \cdot \frac{d}{d x} .
$$

We deduce, after some simple algebra, an alternative definition for the Yukawa coupling:

$$
K(q)=\left(q \cdot \frac{d}{d q}\right)^{2}\left(\frac{y_{2}}{y_{0}}\right)=\frac{W_{1}^{3} \cdot W_{3}}{W_{2}^{3}}=\frac{y_{0}^{3} \cdot W_{3}}{W_{2}^{3}} .
$$

to be compared with the other previous alternative expression previously given (87) for the Yukawa coupling

$$
K(q)=\frac{x(q)^{3} \cdot W_{4}^{1 / 2}}{y_{0}^{2}} \cdot\left(\frac{q}{x(q)} \cdot \frac{d x(q)}{d q}\right)^{3}=\frac{W_{4}^{1 / 2}}{y_{0}^{2}} \cdot\left(q \cdot \frac{d x(q)}{d q}\right)^{3} .
$$

$\ddagger$ For an order-four operator the Wronskian is $W_{4}$. 
In fact from (E.2) we deduce

$$
\left(q \cdot \frac{d x(q)}{d q}\right)^{3}=\frac{W_{1}^{6}}{W_{2}^{3}}=\frac{y_{0}^{6}}{W_{2}^{3}},
$$

and, so, (E.5) is compatible with (E.4) if the following identity is verified:

$$
W_{3}^{2}=W_{4} \cdot y_{0}^{2}=W_{4} \cdot W_{1}^{2} \text {. }
$$

This identity is in fact specific of order-four operators conjugated to their adjoints (see below (E.17)). Therefore we prefer to use definition (E.4) for the Yukawa coupling, instead of the more restricted definition (E.5).

Let us assume that the pullback $p(x)$ has a series expansion of the form

$$
p(x)=\lambda \cdot x^{r} \cdot A(x),
$$

where the exponent $r$ is an integer, where $\lambda$ is a constant, and where $A(x)$ is a function analytic at $x=0$ with the series expansion:

$$
A(x)=1+\alpha_{1} \cdot x+\alpha_{2} \cdot x^{2}+\cdots
$$

The determinantal variables $W_{m}$ transform very nicely under pullbacks $p(x)$ of the form (E.8):

$$
\begin{aligned}
\left(W_{1}(x), W_{2}(x), W_{3}(x), W_{4}(x)\right) & \longrightarrow \\
\left(W_{1}(p(x)), \frac{p^{\prime}}{r} \cdot W_{2}(p(x)), \frac{p^{\prime 3}}{r^{3}} \cdot W_{3}(p(x)), \frac{p^{6}}{r^{6}} \cdot W_{4}(p(x))\right), & p^{\prime}=\frac{d p(x)}{d x} .
\end{aligned}
$$

One can show that the nome (36) of an order- $N$ operator transforms under a pullback $p(x)$ :

$$
q(x) \longrightarrow Q(x) \quad \text { with: } \quad \lambda \cdot Q(x)^{r}=q(p(x)) .
$$

From the covariance property (E.9), and from the previous transformation $q \rightarrow \lambda \cdot q^{r}$ for the nome, one easily gets the transformation of the Yukawa coupling seen as a function of the nome $K(q) \rightarrow K\left(\lambda \cdot q^{r}\right)$ :

$$
\begin{aligned}
K(q(x))= & \frac{W_{1}(x)^{3} \cdot W_{3}(x)}{W_{2}(x)^{3}} \\
& \longrightarrow \quad \frac{W_{1}(p(x))^{3} \cdot W_{3}(p(x))}{W_{2}(p(x))^{3}}=K(q(p(x)))=K\left(\lambda \cdot Q(x)^{r}\right) .
\end{aligned}
$$

For $\lambda=1$ and $r=1$ (i.e. when the pullback is a deformation of the identity transformation), one recovers the known invariance of the Yukawa coupling by pullbacks (see Proposition 3 in [112]).

One finds another pullback invariant ratio, namely:

$$
K^{\star}=\frac{W_{1} \cdot W_{3}^{3}}{W_{4} \cdot W_{2}^{3}}
$$

which is, in fact, nothing but the Yukawa coupling for the adjoint of the original operator.

Another invariance property is worth noting. Let us consider two linear differential operators $\Omega_{1}$ and $\Omega_{2}$ of order $N$ that are equivalent, in the sense of the equivalence of linear differential operators. This means that there exists linear differential operators intertwiners $I_{1}, I_{2}, J_{1}, J_{2}$, of order at most $N-1$ such that

$$
\Omega_{1} \cdot I_{1}=I_{2} \cdot \Omega_{2}, \quad \text { and: } \quad J_{1} \cdot \Omega_{1}=\Omega_{2} \cdot J_{2} .
$$


Let us assume that one of these intertwiners is a linear differential operator of order zero (a function), then the previous homomorphism between operators amounts to saying that the two operators are conjugated by a function:

$$
\Omega_{2}=\rho(x) \cdot \Omega_{1} \cdot \rho(x)^{-1},
$$

which correspond to changing the four solutions as follows: $y_{i} \rightarrow \rho(x) \cdot y_{i}$. In such a case the previous determinant variables transform, again, very nicely under the "gauge" function $\rho(x)$ :

$$
\left(W_{1}, W_{2}, W_{3}, W_{4}\right) \rightarrow\left(\rho(x) \cdot W_{1}, \rho(x)^{2} \cdot W_{2}, \rho(x)^{3} \cdot W_{3}, \rho(x)^{4} \cdot W_{4}\right) .
$$

It is straightforward to see that the Yukawa coupling and the "dual Yukawa", $K^{\star}$, are invariant by such a transformation $\uparrow$. Two conjugated operators (E.14) automatically have the same Yukawa coupling.

Do note that the Yukawa couplings for two operators, which are non trivially homomorphic to each other (intertwiners of order one, two, ...), are actually different. The (pullback invariant) Yukawa coupling is not preserved by operator equivalence (see subsection (8.2)).

Remark: The definition of these determinantal variables $W_{i}$ heavily relies on the MUM structure of the operator between the four solutions the definition of $W_{i}$ 's, in particular the log-ordering of the solutions. It is worth noting that if one permutes the four solution $y_{i}$, one would get 24 other sets of $\left(W_{1}, W_{2}, W_{3}, W_{4}\right)$ which are actually also nicely covariant by pullbacks, thus yielding a finite set of other "Yukawa couplings" or adjoint Yukawa coupling $K^{\star}$ also invariant by pullbacks.

In fact these "Yukawa couplings" (E.4), and other adjoint Yukawa $K^{\star}$ (E.12), can even be defined when the linear differential operator is not MUM, and they are still invariant by pullbacks.

\section{Appendix E.2. Pullback-invariants for higher order ODEs}

These simple calculations can straightforwardly be generalised to higher order linear differential equations. We give here the invariants for higher order linear differential operators.

Let us give, for the $n$-th order linear differential operator the list of the $K_{n}$ invariants by pullback transformations:

$$
\begin{aligned}
& K_{3}=\frac{W_{1}^{3} \cdot W_{3}}{W_{2}^{3}}, \quad K_{4}=\frac{W_{1}^{8} \cdot W_{4}}{W_{2}^{6}}, \quad K_{5}=\frac{W_{1}^{15} \cdot W_{5}}{W_{2}^{10}}, \quad K_{6}=\frac{W_{1}^{24} \cdot W_{6}}{W_{2}^{15}}, \cdots \\
& \left.K_{n}=\frac{W_{1}^{a_{n}} \cdot W_{n}}{W_{2}^{b_{n}}}, \quad \text { with: } \quad a_{n}=n \cdot(n-2), \quad b_{n}=\frac{n \cdot(n-1)}{2} . \quad \text { (E. } 16\right)
\end{aligned}
$$

A $n$-th order linear differential operator has $K_{n}$ as an invariant by pullback transformation, as well as all the $K_{m}$ with $m \leq n . K_{3}$ is the Yukawa coupling, and one remarks, for the order-four operators, that the other pullback invariant $K^{\star}$ (see (E.12)), which is actually also the Yukawa coupling of the adjoint operator, is nothing but $K_{3}^{3} / K_{4}$.

For order-four operators conjugated to their adjoint (see (E.14)) (i.e. operators homomorphic to their adjoint, the intertwiner being an order zero differential operator,

ๆ $K$ and $K^{\star}$ (and their combinations) are the only monomials $W_{1}^{n_{1}} W_{2}^{n_{2}} W_{3}^{n_{3}} W_{4}^{n_{4}}$ to be invariant by (E.9) and (E.15). 
a function), one has the equality

$$
K_{4}=K_{3}^{2}, \quad \text { i.e. } \quad K_{3}=K^{\star}, \quad \text { or } \quad W_{3}^{2}=W_{1}^{2} \cdot W_{4},
$$

to be compared with the equality in Almkvist et al. (see Proposition 2 in [75])

$$
y_{0} y_{3}^{\prime}-y_{3} y_{0}^{\prime}=y_{1} y_{2}^{\prime}-y_{2} y_{1}^{\prime},
$$

which is satisfied when the Calabi-Yau condition that the exterior square is of order five is satisfied.

If a linear differential operator $\Omega_{4}$ verifies condition (E.17), its conjugate by a function, $\rho(x) \cdot \Omega_{4} \cdot \rho(x)^{-1}$, also verifies condition (E.17) (their Yukawa couplings are equal).

The condition (E.17) is not satisfied for linear differential operators homomorphic to their adjoint with non-trivial intertwiner (of order greater than zero). For instance the order-four operator (88) does not satisfy condition (E.17).

\section{Appendix F. More Hadamard products: a Batyrev and van Straten Calabi-Yau ODE}

An order-four operator has been found by Batyrev and van Straten [77] associated with a Calabi-Yau three-fold on $P_{2} \times P_{2}$

$$
\begin{aligned}
B_{1}=\quad \theta^{4} & -3 x \cdot\left(7 \theta^{2}+7 \theta+2\right) \cdot(3 \theta+1) \cdot(3 \theta+2) \\
& -72 x^{2} \cdot(3 \theta+5) \cdot(3 \theta+4) \cdot(3 \theta+2) \cdot(3 \theta+1) .
\end{aligned}
$$

This operator is conjugated to its adjoint: $B_{1} \cdot x=x \cdot \operatorname{adjoint}\left(B_{1}\right)$.

Operator (F.1) is a Calabi-Yau operator [77]: it is MUM, and it is such that its exterior square is of order five. It has a solution analytical at $x=0$ which is actually the Hadamard product of the previous selected hypergeometric ${ }_{2} F_{1}$ :

$$
{ }_{2} F_{1}\left(\left[\frac{1}{3}, \frac{2}{3}\right],[1] ; 27 x\right) \star\left(\frac{1}{1+4 x} \cdot{ }_{2} F_{1}\left(\left[\frac{1}{3}, \frac{2}{3}\right],[1] ; \frac{27 \cdot x}{(1+4 x)^{3}}\right)\right) .
$$

The coefficients of the series expansion of (F.2) are integers: they can actually be written as a sum of product of binomials:

$$
\frac{(3 n) !}{(n !)^{3}} \cdot \sum_{k=0}^{n}\left(\begin{array}{l}
n \\
k
\end{array}\right)^{3}=\left(\begin{array}{c}
3 n \\
n
\end{array}\right) \cdot\left(\begin{array}{c}
2 n \\
n
\end{array}\right) \cdot \sum_{k=0}^{n}\left(\begin{array}{l}
n \\
k
\end{array}\right)^{3} .
$$

The series expansions of the nome and the Yukawa coupling of this Calabi-Yau operator are series with integer coefficients, reading respectively:

$$
\begin{aligned}
& q=x+48 x^{2}+4626 x^{3}+549304 x^{4}+74589735 x^{5}+11014152048 x^{6}+\cdots \\
& K(q)=1+21 q+3861 q^{2}+429159 q^{3}+57224661 q^{4}+7337893896 q^{5}+\cdots \\
& K(x)=1+21 x+4869 x^{2}+896961 x^{3}+175176657 x^{4}+34770008997 x^{5}+\cdots
\end{aligned}
$$




\section{References}

[1] Butera P and Comi M 2002 An on-line library of extended high-temperature expansions of basic observables for the spin- $S$ Ising models on two- and three-dimensional lattices J. Statist. Phys. 109 311-315 URL http://arxiv.org/abs/hep-lat/0204007

[2] Campostrini M, Pelissetto A, Rossi P and Vicari E 2002 25th-order high-temperature expansion results for three-dimensional Ising-like systems on the simple-cubic lattice Phys. Rev. E 65 066127 URL http://arxiv.org/abs/cond-mat/0201180

[3] Arisue H and Fujiwara T 2003 Algorithm of the finite-lattice method for high-temperature expansion of the Ising model in three dimensions Phys. Rev. E 67066109 URL http://arxiv.org/abs/hep-lat/0209002

[4] Bessis J D, Drouffe J M and Moussa P 1976 Positivity constraints for the Ising ferromagnetic model J. Phys. A 92105 URL http://stacks.iop.org/0305-4470/9/i=12/a=015

[5] Eisenstein G 1852 Über eine allgemeine Eigenschaft der Reihen-Entwicklungen aller algebraischen Funktionen Bericht Königl. Preuss. Akad. Wiss. Berlin 441-443

[6] Fatou P 1906 Séries trigonométriques et séries de Taylor Acta Math. 30 335-400 URL http://dx.doi.org/10.1007/BF02418579

[7] Pólya G and Szegö G 1998 Problems and theorems in analysis. II Classics in Mathematics (Berlin: Springer-Verlag) ISBN 3-540-63686-2 Theory of functions, zeros, polynomials, determinants, number theory, geometry

[8] Pólya G 1916 Über Potenzreihen mit ganzzahligen Koeffizienten Math. Ann. 77 497-513 URL http://dx.doi.org/10.1007/BF01456965

[9] Flajolet P, Gerhold S and Salvy B 2005 On the non-holonomic character of logarithms, powers, and the $n$th prime function Electron. J. Combin. 11 Research Paper A2, 16 pp. (electronic) URL http://www.combinatorics.org/ojs/index.php/eljc/article/view/v11i2a2

[10] Carlson F 1921 Über Potenzreihen mit ganzzahligen Koeffizienten Math. Z. 9 1-13 URL http://dx.doi.org/10.1007/BF01378331

[11] Zenine N, Boukraa S, Hassani S and Maillard J-M 2004 The Fuchsian differential equation of the square lattice Ising model $\chi^{(3)}$ susceptibility J. Phys. A 37 9651-9668 URL http://arxiv.org/abs/math-ph/0407060

[12] Boukraa S, Hassani S, Jensen I, Maillard J-M and Zenine N 2010 High-order Fuchsian equations for the square lattice Ising model: $\chi^{(6)}$ J. Phys. A 43 115201, 22 URL http://arxiv.org/abs/0912.4968

[13] Zenine N, Boukraa S, Hassani S and Maillard J-M 2005 Square lattice Ising model susceptibility: connection matrices and singular behaviour of $\chi^{(3)}$ and $\chi^{(4)}$ J. Phys. A 38 9439-9474 URL http://arxiv.org/abs/math-ph/0506065

[14] Bailey D.H, Borwein J.M and Crandall R.E 2006 Integrals of the Ising class, J. Phys. A 39 12271-12302

[15] Boukraa S, Hassani S, Maillard J-M and Zenine N 2007 Singularities of $n$-fold integrals of the Ising class and the theory of elliptic curves J. Phys. A 40 11713-11748 URL http://arxiv.org/abs/0706.3367

[16] Boukraa S, Hassani S, Maillard J-M, McCoy B M and Zenine N 2007 The diagonal Ising susceptibility J. Phys. A 40 8219-8236 URL http://arxiv.org/abs/math-ph/0703009

[17] McCoy B M and Maillard J-M The importance of the Ising model http://arXiv:1203.1456

[18] Boukraa S, Guttmann A J, Hassani S, Jensen I, Maillard J-M, Nickel B and Zenine N 2008 Experimental mathematics on the magnetic susceptibility of the square lattice Ising model J. Phys. A 41 455202, 51 URL http://arxiv.org/abs/0808.0763

[19] Bostan A, Boukraa S, Christol G, Hassani S and Maillard J-M Ising $n$-fold integrals as diagonals of rational functions and integrality of series expansions: integrality versus modularity Preprint http://arxiv.org/abs/1211.6031

[20] Bernšteln I N 1972 Analytic continuation of generalized functions with respect to a parameter Funkcional. Anal. $i$ Priložen. 6 26-40 URL http://www.math.tau.ac.il/ bernstei/Publication_list/publication_texts/Bern-a-cont-FAN.pdf

[21] Kashiwara M 1978 On the holonomic systems of linear differential equations. II Invent. Math. 49 121-135 URL http://dx.doi.org/10.1007/BF01403082

[22] Stanley R P 1980 Differentiably finite power series European J. Combin. 1 175-188

[23] Lipshitz L $1989 \quad D$-finite power series J. Algebra $122 \quad 353-373$ URL http://dx.doi.org/10.1016/0021-8693(89)90222-6

[24] Wu T T, McCoy B M, Tracy C A and Barouch E 1976 Spin-spin correlation functions for the two-dimensional Ising model: Exact theory in the scaling region Phys. Rev. B 13 316-374 URL http://dx.doi.org/10.1103/PhysRevB.13.316 
[25] Zenine N, Boukraa S, Hassani S and Maillard J-M 2005 Ising model susceptibility: the Fuchsian differential equation for $\chi^{(4)}$ and its factorization properties J. Phys. A 38 4149-4173 URL http://arxiv.org/abs/cond-mat/0502155

[26] Bostan A, Boukraa S, Guttmann A J, Hassani S, Jensen I, Maillard J-M and Zenine N 2009 High order Fuchsian equations for the square lattice Ising model: $\tilde{\chi}^{(5)}$ J. Phys. A 42275209 , 32 URL http://arxiv.org/abs/0904.1601

[27] Bostan A, Boukraa S, Hassani S, Maillard J-M, Weil J-A and Zenine N 2009 Globally nilpotent differential operators and the square Ising model J. Phys. A 42 125206, 50 URL http://arxiv.org/abs/0812.4931

[28] André Y 2003 Arithmetic Gevrey series and transcendence. A survey J. Théor. Nombres Bordeaux 15 1-10 Les XXIIèmes Journées Arithmetiques (Lille, 2001) URL http://jtnb.cedram.org/item?id=JTNB_2003_-15_1_1_0

[29] André Y 1989 G-functions and geometry Aspects of Mathematics, E13 (Braunschweig: Friedr. Vieweg \& Sohn) ISBN 3-528-06317-3

[30] Bostan A, Boukraa S, Hassani S, van Hoeij M, Maillard J-M, Weil J-A and Zenine N 2011 The Ising model: from elliptic curves to modular forms and Calabi-Yau equations J. Phys. A 44 045204, 44 URL http://arxiv.org/abs/1007.0535

[31] Assis M, Boukraa S, Hassani S, van Hoeij M, Maillard J-M and McCoy B M 2012 Diagonal Ising susceptibility: elliptic integrals, modular forms and Calabi-Yau equations J. Phys. A 45 075205, 32 URL http://arxiv.org/abs/1110.1705

[32] Yang Y and Zudilin W $2010 \mathrm{On} \mathrm{Sp}_{4}$ modularity of Picard-Fuchs differential equations for Calabi-Yau threefolds Gems in experimental mathematics (Contemp. Math. vol 517) (Providence, RI: Amer. Math. Soc.) pp 381-413 URL http://arxiv.org/abs/0803.3322

[33] Lian B H and Yau S T 1996 Mirror maps, modular relations and hypergeometric series. II Nuclear Phys. B Proc. Suppl. 46 248-262 S-duality and mirror symmetry (Trieste, 1995) URL http://arxiv.org/abs/hep-th/9507153

[34] McKay J and Sebbar A 2007 Replicable functions: an introduction Frontiers in number theory, physics, and geometry. II (Berlin: Springer) pp 373-386 URL http://dx.doi.org/10.1007/978-3-540-30308-4_10

[35] Livné R, Schütt M and Yui N 2010 The modularity of $K 3$ surfaces with non-symplectic group actions Math. Ann. 348 333-355 URL http://arxiv.org/abs/0904.1922

[36] Schütt M 2004 New examples of modular rigid Calabi-Yau threefolds Collect. Math. 55 219-228 URL http://arxiv.org/abs/math/0311106

[37] Gouvêa F Q and Yui N 2011 Rigid Calabi-Yau threefolds over $\mathbb{Q}$ are modular Expo. Math. 29 142-149 URL http://arxiv.org/abs/0902.1466

[38] Saito M H and Yui N 2001 The modularity conjecture for rigid Calabi-Yau threefolds over $Q$ J. Math. Kyoto Univ. 41 403-419 URL http://arxiv.org/abs/math/0009041

[39] van der Poorten A 1978/79 A proof that Euler missed...Apéry's proof of the irrationality of $\zeta(3)$ Math. Intelligencer $\mathbf{1} 195-203$ An informal report URL http://dx.doi.org/10.1007/BF03028234

[40] Glasser M L and Guttmann A J 1994 Lattice Green function (at 0) for the 4D hypercubic lattice J. Phys. A 27 7011-7014 URL http://arxiv.org/abs/cond-mat/9408097

[41] Guttmann A J and Prellberg T 1993 Staircase polygons, elliptic integrals, Heun functions, and lattice Green functions Physical Review E 47 2233-2236 URL http://www. maths. qmul.ac.uk/ tp/papers/pub004.pdf

[42] Joyce G S, Delves R T and Zucker I J 2003 Exact evaluation of the Green functions for the anisotropic face-centred and simple cubic lattices J. Phys. A 36 8661-8672 URL http://dx.doi.org/10.1088/0305-4470/36/32/307

[43] Guttmann A J 2010 Lattice Green's functions in all dimensions J. Phys. A 43 305205, 26 URL http://arxiv.org/abs/1004.1435

[44] Guttmann A J and Rogers M D 2012 Spanning tree generating functions and Mahler measures Preprint http://arxiv.org/abs/1207.2815

[45] Christol G 1990 Globally bounded solutions of differential equations Analytic number theory (Tokyo, 1988) (Lecture Notes in Math. vol 1434) (Berlin: Springer) pp 45-64 URL http://dx.doi.org/10.1007/BFb0097124

[46] Boukraa S, Hassani S and Maillard J-M 2012 Holonomic functions of several complex variables and singularities of anisotropic Ising $n$-fold integrals J. Phys. A: Math. Theor. 45 (2012) 494010 URL http://arxiv.org/abs/1207.1784

[47] Barouch E 1980 On the Ising model in the presence of magnetic field Phys. D 1 333-337 URL http://dx.doi.org/10.1016/0167-2789(80)90031-7

[48] Guttmann A J 2009 Lattice Green functions and Calabi-Yau differential equations J. Phys. A 
42 232001, 6 URL http://dx.doi.org/10.1088/1751-8113/42/23/232001

[49] Roques J 2012 Arithmetic properties of Mirror maps associated with Gauss's hypergeometric equations URL http://www-fourier.ujf-grenoble.fr/ jroques/arith_hypergeo.pdf

[50] Roques J 2012 On generalized hypergeometric equations and mirror maps, Proceedings of the American Mathematical Society, URL http://www-fourier.ujf-grenoble.fr/ jroques/OGHEMM.pdf

[51] Hulek K and Verrill H 2005 On modularity of rigid and nonrigid Calabi-Yau varieties associated to the root lattice $A_{4}$, Nagoya Math. J. 179 103-146

[52] Yui N 2012 Modularity of Calabi-Yau varieties: 2011 and beyond URL http://arXiv: 1212.4308

[53] Adamczewski B and Bell J P Diagonalization and Rationalization of algebraic Laurent series Preprint http://arxiv.org/pdf/1205.4090.pdf

[54] Christol G 1986-1987 Fonctions hypergéométriques bornées Groupe de travail d'analyse ultramétrique $14 \quad 1-16$ URL http://archive.numdam.org/article/GAU_1986-1987_-_14__A4_0.pdf

[55] Pólya G 1921-1922 Sur les séries entières, dont la somme est une fonction algébrique Enseignement Math. $22 \quad 38-47$ URL http://retro.seals.ch/digbib/view?rid=ensmat $-001: 1921-1922: 22:: 220$

[56] Cameron R H and Martin W T 1938 Analytic continuation of diagonals and Hadamard compositions of multiple power series Trans. Amer. Math. Soc. 44 1-7 URL http://dx.doi.org/10.2307/1990100

[57] Hadamard J 1892 Essai sur l'étude des fonctions données par leur développement de Taylor J. Math. Pures Appl. 4 101-186 URL http://portail.mathdoc.fr/JMPA/PDF/JMPA_1892_4_8_A4_0.pdf

[58] Deligne P 1984 Intégration sur un cycle évanescent Invent. Math. 76 129-143 URL http://dx.doi.org/10.1007/BF01388496

[59] Bostan A, Chyzak F, van Hoeij M and Pech L 2011 Explicit formula for the generating series of diagonal 3D rook paths Séminaire Lotharingien de Combinatoire B66a URL http://www.emis.de/journals/SLC/wpapers/s66bochhope.html

[60] Christol G 1984 Diagonales de fractions rationnelles et équations différentielles Study group on ultrametric analysis, 10th year: 1982/83, No. 2, Exp. No. 18 (Paris: Inst. Henri Poincaré) pp 1-10 URL http://archive.numdam.org/article/GAU_1982-1983__10_2_A4_0.pdf

[61] Christol G 1985 Diagonales de fractions rationnelles et équations de Picard-Fuchs Study group on ultrametric analysis, 12th year, 1984/85, No. 1, Exp. No. 13 (Paris: Secrétariat Math.) pp 1-12 URL http://archive.numdam.org/article/GAU_1984-1985__12_1_A8_0.pdf

[62] Christol G 1988 Diagonales de fractions rationnelles Séminaire de Théorie des Nombres, Paris 1986-87 (Progr. Math. vol 75) (Boston, MA: Birkhäuser Boston) pp 65-90

[63] Lipshitz L 1988 The diagonal of a D-finite power series is D-finite $J$. Algebra 113 373-378 URL http://dx.doi.org/10.1016/0021-8693(88)90166-4

[64] Lipshitz L and van der Poorten A J 1990 Rational functions, diagonals, automata and arithmetic Number theory (Banff, AB, 1988) (Berlin: de Gruyter) pp 339-358

[65] Furstenberg H 1967 Algebraic functions over finite fields J. Algebra 7 271-277 URL http://dx.doi.org/10.1016/0021-8693(67)90061-0

[66] Ihara Y 1974 Schwarzian equations J. Fac. Sci. Univ. Tokyo Sect. IA Math. 21 97-118 URL http://repository.dl.itc.u-tokyo.ac.jp/dspace/bitstream/2261/6446/1/jfs210106.pdf

[67] Honda T 1981 Algebraic differential equations Symposia Mathematica, Vol. XXIV (Sympos., INDAM, Rome, 1979) (London: Academic Press) pp 169-204

[68] Koike M 1999 Hypergeometric polynomials over finite fields Tohoku Math. J. (2) 51 75-79 URL http://dx.doi.org/10.2748/tmj/1178224854

[69] Necer A 1997 Séries formelles et produit de Hadamard J. Théor. Nombres Bordeaux 9 319-335 URL http://archive.numdam.org/article/JTNB_1997__9_2_319_0.pdf

[70] Hurwitz A 1898 Sur un théorème de M. Hadamard C. R. Math. Acad. Sci. Paris 128 350-353

[71] Denef J and Lipshitz L 1987 Algebraic power series and diagonals J. Number Theory 26 46-67 URL http://dx.doi.org/10.1016/0022-314X(87)90095-3

[72] Boukraa S, Hassani S, Maillard J-M and Zenine N 2007 Landau singularities and singularities of holonomic integrals of the Ising class J. Phys. A 40 2583-2614 URL http://arxiv.org/abs/math-ph/0701016

[73] Sathaye A 1983 Generalized Newton-Puiseux expansion and Abhyankar-Moh semigroup theorem Invent. Math. 74 149-157 URL http://dx.doi.org/10.1007/BF01388535

[74] Aparicio-Monforte A and Kauers M 2012 Formal Laurent series in several variables Expo. Math. To appear URL http://www.risc.jku.at/people/mkauers/publications/aparicio12.pdf 
[75] Almkvist G and Zudilin W 2006 Differential equations, mirror maps and zeta values Mirror symmetry. V (AMS/IP Stud. Adv. Math. vol 38) (Providence, RI: Amer. Math. Soc.) pp 481-515 URL http://arxiv.org/abs/math/0402386

[76] Morrison D R 1993 Mirror symmetry and rational curves on quintic threefolds: a guide for mathematicians J. Amer. Math. Soc. 6 223-247 URL http://arxiv.org/abs/alg-geom/9202004

[77] Batyrev V V and van Straten D 1995 Generalized hypergeometric functions and rational curves on Calabi-Yau complete intersections in toric varieties Comm. Math. Phys. 168 493-533 URL http://arxiv.org/abs/alg-geom/9307010

[78] Almkvist G, van Enckevort C, van Straten D and Zudilin W Tables of CalabiYau equations Preprint http://arxiv.org/pdf/math/0507430 and database http://enriques.mathematik. uni-mainz.de/CYequations/

[79] Chen Y H, Yang Y and Yui N 2008 Monodromy of Picard-Fuchs differential equations for Calabi-Yau threefolds J. Reine Angew. Math. 616 167-203 With an appendix by Cord Erdenberger URL http://arxiv.org/abs/math/0605675

[80] André Y 2004 Sur la conjecture des p-courbures de Grothendieck-Katz et un problème de Dwork Geometric aspects of Dwork theory. Vol. I, II (Walter de Gruyter GmbH \& Co. KG, Berlin) pp 55-112

[81] Almkvist G 2006 Calabi-Yau differential equations of degree 2 and 3 and Yifan Yang's pullback Preprint http://arxiv.org/abs/math/0612215

[82] Almkvist G 2007 Binomial identities related to Calabi-Yau differential equations Preprint http://arxiv.org/abs/math/0703255

[83] Zagier D 2009 Integral solutions of Apéry-like recurrence equations Groups and symmetries (CRM Proc. Lecture Notes vol 47) (Providence, RI: Amer. Math. Soc.) pp 349-366 URL http://people.mpim-bonn.mpg.de/zagier/files/tex/AperylikeRecEqs/fulltext.pdf

[84] Egorychev G P 1984 Integral representation and the computation of combinatorial sums (Translations of Mathematical Monographs vol 59) (Providence, RI: American Mathematical Society) ISBN 0-8218-4512-8 Translated from the Russian by H. H. McFadden, Translation edited by Lev J. Leifman

[85] Strehl V 1994 Binomial identities-combinatorial and algorithmic aspects Discrete Math. 136 309-346 Trends in discrete mathematics URL http://dx.doi.org/10.1016/0012-365X(94)00118-3

[86] Schmidt A L 1995 Legendre transforms and Apéry's sequences J. Austral. Math. Soc. Ser. A 58 358-375 URL http://dx.doi.org/10.1017/S1446788700038350

[87] Zudilin W 2004 On a combinatorial problem of Asmus Schmidt Electron. J. Combin. $\mathbf{1 1}$ Research Paper 22, 8 pp. (electronic) URL http://www.combinatorics.org/Volume_11/Abstracts/v11i1r22.html

[88] Nickel B, Jensen I, Boukraa S, Guttmann A J, Hassani S, Maillard J-M and Zenine N 2010 Square lattice Ising model $\tilde{\chi}^{(5)}$ ODE in exact arithmetic J. Phys. A 43 195205, 24 URL http://arxiv.org/abs/1002.0161

[89] Dwork B, Gerotto G and Sullivan F J 1994 An introduction to G-functions (Annals of Mathematics Studies vol 133) (Princeton, NJ: Princeton University Press) ISBN 0-69103681-0

[90] Katz N M 1972 Algebraic solutions of differential equations ( $p$-curvature and the Hodge filtration) Invent. Math. 18 1-118 URL http://dx.doi.org/10.1007/BF01389714

[91] Beukers F and Heckman G 1989 Monodromy for the hypergeometric function ${ }_{n} F_{n-1}$ Invent. Math. 95 325-354 URL http://dx.doi.org/10.1007/BF01393900

[92] van Hoeij M and Weil J-A 2005 Solving second order linear differential equations with Klein's theorem Proceedings of the 2005 international symposium on Symbolic and algebraic computation ISSAC'05 (New York, NY, USA: ACM) pp 340-347 ISBN 1-59593-095-7 URL http://doi.acm.org/10.1145/1073884.1073931

[93] Bostan A, Boukraa S, Hassani S, Maillard J-M, Weil J-A, Zenine N and Abarenkova N 2010 Renormalization, isogenies, and rational symmetries of differential equations Adv. Math. Phys. Art. ID 941560, 44 URL http://arxiv.org/abs/0911.5466

[94] Golyshev V and Stienstra J 2007 Fuchsian equations of type DN Commun. Number Theory Phys. 1 323-346 URL http://arxiv.org/abs/math/0701936

[95] Lian B H and Yau S T 1996 Arithmetic properties of mirror map and quantum coupling Comm. Math. Phys. 176 163-191 URL http://arxiv.org/abs/hep-th/9411234

[96] Maier R S 2009 On rationally parametrized modular equations J. Ramanujan Math. Soc. 24 1-73 URL http://arxiv.org/abs/math/0611041

[97] Hibino $\mathrm{T}$ and Murabayashi N 1997 Modular equations of hyperelliptic $X_{0}(N)$ and an application Acta Arith. 82 279-291 URL 
http: //citeseerx . ist.psu.edu/viewdoc/download?doi=10.1.1.113.3530\&rep=rep1\&type=pdf

[98] Delaygue E 2011 Propriétés arithmétiques des applications miroir Ph.D. thesis Université de Grenoble URL http://tel.archives-ouvertes.fr/tel-00628016/

[99] Krattenthaler C and Rivoal T 2010 On the integrality of the Taylor coefficients of mirror maps Duke Math. J. 151 175-218 URL http://arxiv.org/abs/0907.2577

[100] Manin Y I 1999 Frobenius manifolds, quantum cohomology, and moduli spaces (American Mathematical Society Colloquium Publications vol 47) (Providence, RI: American Mathematical Society) ISBN 0-8218-1917-8

[101] Katz N M 1990 Exponential sums and differential equations (Annals of Mathematics Studies vol 124) (Princeton, NJ: Princeton University Press) ISBN 0-691-08598-6; 0-691-08599-4

[102] McKean H and Moll V 1997 Elliptic curves (Cambridge: Cambridge University Press) ISBN 0-521-58228-8; 0-521-65817-9 Function theory, geometry, arithmetic

[103] Husemöller D 2004 Elliptic curves 2nd ed (Graduate Texts in Mathematics vol 111) (New York: Springer-Verlag) ISBN 0-387-95490-2 With appendices by Otto Forster, Ruth Lawrence and Stefan Theisen

[104] Bouw I I and Möller M 2010 Differential equations associated with nonarithmetic Fuchsian groups J. Lond. Math. Soc. (2) 81 65-90 URL http://arxiv.org/abs/0710.5277

[105] Dettweiler M and Reiter S 2010 On globally nilpotent differential equations J. Differential Equations 248 2736-2745 URL http://arxiv.org/abs/math/0605383

[106] Chazy J 1910 Sur les équations différentielles dont l'intégrale générale possède une coupure essentielle mobile C. R. Acad. Sc. Paris, 150, 456-458

[107] Harnad J and McKay J 2000 Modular solutions to equations of generalized Halphen type em Proceedings: Mathematical, Physical and Engineering Sciences published by the Royal Society, 456 261-294 URL http://arXiv:solv-int/9804006

[108] Várilly-Alvarado A and Zywina D 2009 Arithmetic $E_{8}$ lattices with maximal Galois action LMS J. Comput. Math. 12 144-165 URL http://arxiv.org/abs/0803.3063

[109] Rodriguez-Villegas F Integral ratios of factorials and algebraic hypergeometric functions Preprint http://www.math.utexas.edu/users/villegas/publications/oberwolfach-05.pdf (Summary of talk at Oberwolfach 2005)

[110] Chebyshev P L 1852 Mémoire sur les nombres premiers J. de Math. Pures Appl. 17 366-390 URL http://archive.numdam.org/article/ASENS_1920_3_37__1_0.pdf

[111] Bober J W 2009 Factorial ratios, hypergeometric series, and a family of step functions J. Lond. Math. Soc. (2) 79 422-444 URL http://arxiv.org/abs/0709.1977

[112] Almkvist G, van Straten D and Zudilin W 2011 Generalizations of Clausen's formula and algebraic transformations of Calabi-Yau differential equations Proc. Edinb. Math. Soc. (2) 54 273-295 URL http://dx.doi.org/10.1017/S0013091509000959 